\title{
Adaptive Modified Function Projective Lag Synchronization of Uncertain Hyperchaotic Dynamical Systems with the Same or Different Dimension and Structure
}

\author{
Xiuli Chai, ${ }^{1}$ Zhihua Gan, ${ }^{2}$ and Chunxiao Shi ${ }^{1}$ \\ ${ }^{1}$ College of Computer and Information Engineering, Institute of Image Processing and Pattern Recognition, \\ Henan University, Kaifeng 475004, China \\ ${ }^{2}$ Department of Software, Institute of Intelligent Network System, Henan University, Kaifeng 475004, China \\ Correspondence should be addressed to Xiuli Chai; chaixiuli@henu.edu.cn
}

Received 29 April 2013; Revised 22 May 2013; Accepted 22 May 2013

Academic Editor: Wang Xing-yuan

Copyright (C) 2013 Xiuli Chai et al. This is an open access article distributed under the Creative Commons Attribution License, which permits unrestricted use, distribution, and reproduction in any medium, provided the original work is properly cited.

\begin{abstract}
Modified function projective lag synchronization (MFPLS) of uncertain hyperchaotic dynamical systems with the same or different dimensions and structures is studied. Based on Lyapunov stability theory, a general theorem for controller designing, parameter update rule designing, and control gain strength adapt law designing is introduced by using adaptive control method. Furthermore, the scheme is applied to four typical examples: MFPLS between two five-dimensional hyperchaotic systems with the same structures, MFPLS between two four-dimensional hyperchaotic systems with different structures, MFPLS between a four-dimensional hyperchaotic system and a three-dimensional chaotic system and MFPLS between a novel three-dimensional chaotic system, and a five-dimensional hyperchaotic system. And the system parameters are all uncertain. Corresponding numerical simulations are performed to verify and illustrate the analytical results.
\end{abstract}

\section{Introduction}

During the past three decades, chaos synchronization has been a hot topic in nonlinear science due to its various applications [1-3]. A variety of synchronization approaches have been revealed, such as complete synchronization (CS) [4], antisynchronization (AS) [5], phase synchronization [6], lag synchronization (LS) [7], projective synchronization (PS) [8], function projective synchronization (FPS) [9-11], and others $[12,13]$. Recently, modified function projective synchronization (MFPS) is proposed, in which the drive system and the response system could be synchronized up to a scaling function matrix [14]. MFPS can enhance the security of communication, because the unpredictability of the scaling functions increases the complexity of the systems. So, MFPS attracts the interests of researchers in many fields [15-20].

In the past, many theoretical results focused on the systems as identical, similar. But in a great many practical situations, the parameters of systems cannot be known or certain entirely, and sometimes the synchronization is carried out even though the drive system and the response system have different dimensions, especially the systems in biological science and social science. Recently, some researchers carried out works related to this.

Sun et al. [21] proposed the MFPS of uncertain hyperchaotic systems with identical or nonidentical structures, gave a general formula for designing the controllers and parameter update rules, and applied the theoretical results to three typical cases. Zheng [22] investigated the MFPS between two different dimensional chaotic systems with fully unknown or partially unknown parameters via increased order method, designed a unified adaptive controller and parameter update laws, and the control strength of the controller can adaptively be identified. Reference [23] studied the MFPS between fourdimensional Lorenz and Chen hyperchaotic dynamical systems with fully unknown parameters; scaling function matrix had the form of $\mathbf{M} h(t), \mathbf{M}$ is a constant diagonal matrix and $h(t)$ is a continuous differentiable function with $h(t) \neq 0$, and the scaling function matrix is more flexible and variable through choosing different $\mathbf{M}$ and $h(t)$. So, it has broad application prospects in practical situations [24]. 
Time delay is frequently encountered in the controlling process of many nonlinear systems, the existence of which makes the controller design and analysis much more complex. Some existing papers consider the influence of time delay on MFPS of chaotic systems. Du et al. [25] introduced modified function projective lag synchronization (MFPLS) and investigated a general method of MFPLS based on Lyapunov stability theory. But in the analysis, they suppose that the system parameters were known and the dimensions of the drive system and response system were equal. Recently, Cai et al. [26] studied the modified function lag projective synchronization of a financial hyperchaotic system, when the parameters are known and unknown, respectively. And their work aimed for MFPLS of the concrete system and could not give the general method. More recently, Liu et al. [27] analyzed the MFPLS between two nonidentical multiscroll chaotic systems with unknown disturbances. Du [28] researched the MFPS of complex dynamical networks with different nodes through adaptive open-plus-closed-loop control method and investigated MFPS in drive-response dynamical networks with time-varying coupling delay.

Inspired by the previous discussion, in this paper, we study the modified function projective lag synchronization (MFPLS) of hyperchaotic systems; the parameters of the systems are all uncertain, dimensions and structures of the drive system and the response system are the same or different. Based on the Lyapunov stability theory and adaptive control technique, an adaptive controller and corresponding parameter update rule are constructed. By applying the method, MFPLS between a five-dimensional hyperchaotic system and itself with uncertain parameters, a four-dimensional hyperchaotic system and another four-dimensional hyperchaotic system with unknown parameters and different structures, a four-dimensional hyperchaotic system and a threedimensional chaotic system with uncertain parameters, and a three-dimensional chaotic system and a five-dimensional hyperchaotic system with unknown parameters is achieved. Corresponding simulation results show the effectiveness of the proposed scheme.

The outline of the paper is as follows. In Section 2, the definition of MFPLS is introduced. In Section 3, a general method for MFPLS between two uncertain hyperchaotic systems with the same or different dimension and structure is given. Based on the Lyapunov stability theory and adaptive control technique, an adaptive controller, corresponding parameter update rule, and control gain strength adapt law are designed. In Section 4, we give four typical examples to verify the effectiveness of the proposed scheme by numerical simulation. Finally, the conclusions are drawn in Section 5.

\section{The Definition of MFPLS}

Consider the drive (master) chaotic system and the response (slave) chaotic system given in the following form:

$$
\begin{gathered}
\dot{\mathbf{x}}(t)=f(\mathbf{x}(t)), \\
\dot{\mathbf{y}}(t)=g(\mathbf{y}(t))+\mathbf{U}(t),
\end{gathered}
$$

where $\mathbf{x}(t), \mathbf{y}(t)$ are the state vectors of systems (1) and (2), respectively; $f, g$ are two continuous vector functions, and $\mathbf{U}$ is a controller to be designed for synchronization between systems (1) and (2). The error dynamical system is defined as follows:

$$
\mathbf{e}(t)=\mathbf{y}(t)-\mathbf{M} h(t) \mathbf{x}(t-\boldsymbol{\tau}),
$$

where $\mathbf{M}$ is a constant diagonal matrix, $h(t)$ is a nonzero continuous differentiable function, and the time delay vector is $\boldsymbol{\tau}$.

Definition 1. If there exists a constant diagonal matrix $\mathbf{M}$ and function $h(t)$, such that $\lim _{t \rightarrow \infty}\|\mathbf{e}(t)\|=0$, then the synchronization between the system (1) and system (2) is modified function projective lag synchronization (MFPLS).

Remark 2. If $h(t)=1$ and $\boldsymbol{\tau}=0$, MFPLS becomes modified projective synchronization. If $\mathbf{M}=1$ and $\boldsymbol{\tau}=0$, MFPLS turns out to be the function projective synchronization. If $\boldsymbol{\tau}=0$, MFPLS is MFPS. If $\mathbf{M}=1$ and $h(t)=1$, then lag synchronization appears.

\section{The Modified Function Projective Lag Synchronization Scheme}

Suppose that the parameters in the drive and response system are uncertain. Rewrite them as follows:

$$
\begin{gathered}
\dot{\mathbf{x}}(t)=f_{1}(\mathbf{x}(t)) \mathbf{A}+f_{2}(\mathbf{x}(t)), \\
\dot{\mathbf{y}}(t)=g_{1}(\mathbf{y}(t)) \mathbf{B}+g_{2}(\mathbf{y}(t))+\mathbf{U}(t),
\end{gathered}
$$

where $\mathbf{x}(t)=\left(x_{1}, x_{2}, \ldots, x_{m}\right)^{\mathrm{T}} \in R^{m}, \mathbf{y}(t)=\left(y_{1}, y_{2}, \ldots\right.$, $\left.y_{n}\right)^{\mathrm{T}} \in R^{n}$ are the state vectors of systems (4) and (5), respectively; $f: R^{m} \rightarrow R^{m}, g: R^{n} \rightarrow R^{n}$ are two continuous vector functions; $\mathbf{A}, \mathbf{B}$ are the uncertain parameter vectors of the drive and response system, respectively; $\mathbf{U}$ is a controller to be designed for synchronization between systems (4) and (5). The error system is defined as (3).

The dimension of the drive system is $m$ and the order of the response system is $n$; they are not mostly the same. So, in the following, we discuss three cases: $m=n, m>n$, and $n>m$ and give a general scheme for the synchronization controller design and parameter update rule design.

Case $1(m=n)$. At this time, the drive and response systems are of the same order.

Theorem 3. For the given diagonal matrix $\mathbf{M}=\operatorname{diag}\left\{m_{1}, m_{2}\right.$, $\left.\ldots, m_{n}\right\} \in R^{n \times n}$, nonzero continuous differentiable function $\mathbf{h}(t)$, and time delay vector $\boldsymbol{\tau}=\left(\tau_{1}, \tau_{2}, \ldots, \tau_{n}\right)^{T}$, the modified function projective lag synchronization (MFPLS) between system (4) and system (5) is achieved by the following controller (6), the control gain matrix $\mathbf{K}=\operatorname{diag}\left(k_{1}, k_{2}, \ldots, k_{n}\right)$, $\left(k_{i}\right.$ is 
the control gain strength) (7), the parameter update laws (8) and (9) as below:

$$
\begin{gathered}
\mathbf{U}(t)=-g_{1}(\mathbf{y}(t)) \widehat{\mathbf{B}}-g_{2}(\mathbf{y}(t))+\mathbf{M} \dot{h}(t) \mathbf{x}(t-\boldsymbol{\tau}) \\
+\mathbf{M} h(t)\left(f_{1}(\mathbf{x}(t)) \widehat{\mathbf{A}}+f_{2}(\mathbf{x}(t))\right)-\mathbf{K e}, \\
\dot{k}_{i}=\varepsilon_{i} e_{i}^{2}, \quad \varepsilon_{i}>0, i=1,2, \ldots, n, \\
\dot{\mathbf{e}}_{A}(t)=-f_{1}^{T}(\mathbf{x}(t)) h^{T}(t) \mathbf{M e}(t), \\
\dot{\mathbf{e}}_{B}(t)=g_{1}(\mathbf{y}(t)) \mathbf{e}(t),
\end{gathered}
$$

where $\mathbf{e}_{i}(t)=y_{i}(t)-m_{i} h(t) x_{i}\left(t-\tau_{i}\right) ; \widehat{\mathbf{A}}, \widehat{\mathbf{B}}$ are the estimated values of unknown parameters $\mathbf{A}$ and $\mathbf{B}$, respectively; $\mathbf{e}_{A}(t)=$ $\widehat{\mathbf{A}}-\mathbf{A}$ and $\mathbf{e}_{B}(t)=\widehat{\mathbf{B}}-\mathbf{B}$ are parameter error vector matrices.

Proof. According to the definition of MFPLS, the error vector has the following form: $\mathbf{e}(t)=\mathbf{y}(t)-\mathbf{M} h(t) \mathbf{x}(t-\boldsymbol{\tau})$. And the error dynamical system is

$$
\begin{aligned}
\dot{\mathbf{e}}(t)= & \dot{\mathbf{y}}(t)-\mathbf{M} \dot{h}(t) \mathbf{x}(t-\boldsymbol{\tau})-\mathbf{M} h(t) \dot{\mathbf{x}}(t-\boldsymbol{\tau}) \\
= & g_{1}(\mathbf{y}(t)) \mathbf{B}+g_{2}(\mathbf{y}(t))+\mathbf{U}(t)-\mathbf{M} \dot{h}(t) \mathbf{x}(t-\boldsymbol{\tau}) \\
& -\mathbf{M} h(t)\left(f_{1}(\mathbf{x}(t)) \mathbf{A}+f_{2}(\mathbf{x}(t))\right) .
\end{aligned}
$$

By substituting (6) into (10), one can obtain the following:

$$
\begin{aligned}
\dot{\mathbf{e}}(t) & =g_{1}(\mathbf{y}(t))(\mathbf{B}-\widehat{\mathbf{B}})+\mathbf{M} h(t) f_{1}(\mathbf{x}(t))(\widehat{\mathbf{A}}-\mathbf{A})-\mathbf{K e} \\
& =-g_{1}(\mathbf{y}(t)) \mathbf{e}_{B}(t)+\mathbf{M} h(t) f_{1}(\mathbf{x}(t)) \mathbf{e}_{A}(t)-\mathbf{K e} .
\end{aligned}
$$

The Lyapunov function is chosen as

$$
\begin{aligned}
V(t)= & \frac{1}{2}\left(\mathbf{e}^{\mathrm{T}}(t) \mathbf{e}(t)+\mathbf{e}_{A}^{\mathrm{T}}(t) \mathbf{e}_{A}(t)+\mathbf{e}_{B}^{\mathrm{T}}(t) \mathbf{e}_{B}(t)\right) \\
& +\frac{1}{2} \sum_{i=1}^{n} \frac{1}{\varepsilon_{i}}\left(k_{i}-k\right)^{2},
\end{aligned}
$$

where $k>0$ is a positive constant.

Then the time derivation of the Lyapunov function along the trajectory of error systems (10) is

$$
\begin{aligned}
\dot{V}(t)= & \mathbf{e}^{\mathrm{T}}(t) \dot{\mathbf{e}}(t)+\mathbf{e}_{A}^{\mathrm{T}}(t) \dot{\mathbf{e}}_{A}(t)+\mathbf{e}_{B}^{\mathrm{T}}(t) \dot{\mathbf{e}}_{B}(t) \\
& +\dot{k}_{i} \sum_{i=1}^{n} \frac{1}{\varepsilon_{i}}\left(k_{i}-k\right)^{2} .
\end{aligned}
$$

Constituting (7), (8), and (9) into (13), we get the following:

$$
\begin{aligned}
\dot{V}(t)= & \mathbf{e}^{\mathrm{T}}(t) \dot{\mathbf{e}}(t)+\mathbf{e}_{A}^{\mathrm{T}}(t) \dot{\mathbf{e}}_{A}(t)+\mathbf{e}_{B}^{\mathrm{T}}(t) \dot{\mathbf{e}}_{B}(t) \\
& +\dot{k}_{i} \sum_{i=1}^{n} \frac{1}{\varepsilon_{i}}\left(k_{i}-k\right)^{2}
\end{aligned}
$$

$$
\begin{aligned}
= & -\mathbf{e}^{\mathrm{T}}(t) g_{1}(\mathbf{y}(t)) \mathbf{e}_{B}(t)+\mathbf{e}^{\mathrm{T}}(t) \mathbf{M} h(t) f_{1}(\mathbf{x}(t)) \mathbf{e}_{A}(t) \\
& -\mathbf{e}^{\mathrm{T}}(t) \mathbf{K} \mathbf{e}+\sum_{i=1}^{n}\left(k_{i}-k\right)^{2} e_{i}^{2} \\
= & -k \mathbf{e}^{\mathrm{T}}(t) \mathbf{e}<0 .
\end{aligned}
$$

$V(t)$ is positive definite and $\dot{V}(t)<0$; thus, according to Barbalat's Lemma, MFPLS between the drive system (4) and the response system (5) is achieved. However, we cannot conclude that the unknown parameters can be estimated to their true values.

Linear Independence Condition. To achieve synchronizationbased parameter identification, the nonlinear vector functions $-f_{1}^{\mathrm{T}}(\mathbf{x}(t)) h^{\mathrm{T}}(t) \mathbf{M}$ and $g_{1}^{\mathrm{T}}(\mathbf{y}(t))$ must be linearly independent of the synchronization manifold, and then the unknown parameters can be identified $[29,30]$.

Remark 4. Suppose that the parameter $\mathbf{A}$ in the drive system (4) are known in priori, then the controller and parameter update rule can be designed as follows:

$$
\begin{gathered}
\mathbf{U}(t)=-g_{1}(\mathbf{y}(t)) \widehat{\mathbf{B}}-g_{2}(\mathbf{y}(t))+\mathbf{M} \dot{h}(t) \mathbf{x}(t-\boldsymbol{\tau}) \\
+\mathbf{M} h(t)\left(f_{1}(\mathbf{x}(t)) A+f_{2}(\mathbf{x}(t))\right)-\mathbf{K e}, \\
\dot{\mathbf{e}}_{B}(t)=g_{1}(\mathbf{y}(t)) \mathbf{e}(t) .
\end{gathered}
$$

Control gain strength adapt rule is as (7).

Remark 5. Suppose that the parameter $\mathbf{B}$ in the response system (5) is known in priori, then the controller and parameter update rule can be modified as follows:

$$
\begin{aligned}
\mathbf{U}(t)= & -g_{1}(\mathbf{y}(t)) \mathbf{B}-g_{2}(\mathbf{y}(t))+\mathbf{M} \dot{h}(t) \mathbf{x}(t-\boldsymbol{\tau}) \\
& +\mathbf{M} h(t)\left(f_{1}(\mathbf{x}(t)) \widehat{\mathbf{A}}+f_{2}(\mathbf{x}(t))\right)-\mathbf{K e}, \\
& \dot{\mathbf{e}}_{A}(t)=-f_{1}^{\mathrm{T}}(\mathbf{x}(t)) h^{\mathrm{T}}(t) \mathbf{M e}(t) .
\end{aligned}
$$

Control gain strength adapt law is as (7).

Remark 6. If the drive and response system have the identical structures, then the controller and parameter update rule is as follows:

$$
\begin{aligned}
\mathbf{U}(t)= & \left(-f_{1}(\mathbf{y}(t))+\mathbf{M} h(t) f_{1}(\mathbf{x}(t))\right) \widehat{\mathbf{A}} \\
& +\left(-f_{2}(\mathbf{y}(t))+\mathbf{M} h(t) f_{2}(\mathbf{x}(t))\right) \\
& +\mathbf{M} \dot{h}(t) \mathbf{x}(t-\boldsymbol{\tau})-\mathbf{K e}, \\
\dot{\mathbf{e}}_{A}(t) & =-f_{1}^{\mathrm{T}}(\mathbf{x}(t)) h^{\mathrm{T}}(t) \mathbf{M e}(t) .
\end{aligned}
$$

Control gain strength adapt rule is as (7). When the drive system and the response system have the concrete structure, the controller can be largely simplified.

Remark 7. In many references [25-28], the control gain strength is fixed, and sometimes it may be the maximal; thus, it can give a kind of energy waste. The method of our paper 
is different from them. The control gain strength $k_{i}$ can be automatically adapted to a suitable value depending on the initial values.

Case $2(m>n)$. The dimension of the drive system is higher than that of the response system. And we can achieve MFPLS through adding extra-auxiliary states to the response system, so that the dimensions of the drive and response system are equal.

Denote the auxiliary states as $y^{\prime} \in R^{m-n}$, and the auxiliary vector is as follow:

$$
\begin{aligned}
\dot{y}^{\prime} & =\left(\phi_{1}(\mathbf{y}), \phi_{2}(\mathbf{y}), \ldots, \phi_{m-n}(\mathbf{y})\right)+U^{\prime}(t) \\
& =g_{1}^{\prime}(\mathbf{y}(t)) \mathbf{B}+g_{2}^{\prime}(\mathbf{y}(t))+U^{\prime}(t)
\end{aligned}
$$

Then, the response system is modified as follows:

$$
\dot{\tilde{\mathbf{y}}}(t)=g_{1}(\widetilde{\mathbf{y}}(t)) \mathbf{B}+g_{2}(\widetilde{\mathbf{y}}(t))+\widetilde{\mathbf{U}}(t)
$$

where $\widetilde{\mathbf{y}}=\left(\begin{array}{c}\mathbf{y} \\ \mathbf{y}^{\prime}\end{array}\right), g_{1}(\widetilde{\mathbf{y}}(t))=\left(\begin{array}{c}g_{1}(\mathbf{y}(t)) \\ g_{1}^{\prime}(\mathbf{y}(t))\end{array}\right), g_{2}(\widetilde{\mathbf{y}}(t))=\left(\begin{array}{c}g_{2}(\mathbf{y}(t)) \\ g_{2}^{\prime}(\mathbf{y}(t))\end{array}\right)$, $\widetilde{\mathbf{U}}(t)=\left(\begin{array}{c}\mathbf{U}(t) \\ \mathbf{U}^{\prime}(t)\end{array}\right)$.

Thus the controller, parameter update law, and control gain strength adapt rule are designed as follows:

$$
\begin{gathered}
\widetilde{\mathbf{U}}(t)=-g_{1}(\widetilde{\mathbf{y}}(t)) \widehat{\mathbf{B}}-g_{2}(\widetilde{\mathbf{y}}(t))+\mathbf{M} \dot{h}(t) \mathbf{x}(t-\boldsymbol{\tau}) \\
+\mathbf{M} h(t)\left(f_{1}(\mathbf{x}(t)) \widehat{\mathbf{A}}+f_{2}(\mathbf{x}(t))\right)-\mathbf{K e}, \\
\dot{k}_{i}=\varepsilon_{i} e_{i}^{2}, \quad \varepsilon_{i}>0, i=1,2, \ldots, m, \\
\dot{\mathbf{e}}_{A}(t)=-f_{1}^{\mathrm{T}}(\mathbf{x}(t)) h^{\mathrm{T}}(t) \mathbf{M e}(t), \\
\dot{\mathbf{e}}_{B}(t)=g_{1}(\widetilde{\mathbf{y}}(t)) \mathbf{e}(t) .
\end{gathered}
$$

The proof is similar to Case 1 and is omitted here.

Case $3(n>m)$. The dimension of the drive system is lower than that of the response system. We can achieve the MFPLS in increased order method. This time, we add some auxiliary states which is the function of drive state $\mathbf{x}$ to the drive system, and in the final, the order of the drive system is equal to that of the response system.

Denote the auxiliary states as $\mathbf{x}^{\prime} \in R^{n-m}$, and the auxiliary function vector is

$$
\begin{aligned}
\dot{\mathbf{x}}^{\prime} & =\boldsymbol{\varphi}(\mathbf{x})=\left(\varphi_{1}(\mathbf{x}), \varphi_{2}(\mathbf{x}), \ldots, \varphi_{n-m}(\mathbf{x})\right) \\
& =f_{1}^{\prime}(\mathbf{x}(t)) \mathbf{A}+f_{2}^{\prime}(\mathbf{x}(t)) \in R^{n-m} .
\end{aligned}
$$

Then, the drive system is composed as

$$
\widetilde{\mathbf{x}}(t)=f_{1}(\widetilde{\mathbf{x}}(t)) \mathbf{A}+f_{2}(\widetilde{\mathbf{x}}(t)),
$$

where $\widetilde{\mathbf{x}}=\left(\begin{array}{c}\mathbf{x} \\ \mathbf{x}^{\prime}\end{array}\right), f_{1}(\widetilde{\mathbf{x}}(t))=\left(\begin{array}{c}f_{1}(\mathbf{x}(t)) \\ f_{1}^{\prime}(\mathbf{x}(t))\end{array}\right), f_{2}(\widetilde{\mathbf{x}}(t))=\left(\begin{array}{c}f_{2}(\mathbf{x}(t)) \\ f_{2}^{\prime}(\mathbf{x}(t))\end{array}\right)$.
The controller, parameter update law, and control gain strength adapt rule are illustrated as follows:

$$
\begin{gathered}
\mathbf{U}(t)=-g_{1}(\mathbf{y}(t)) \widehat{\mathbf{B}}-g_{2}(\mathbf{y}(t))+\mathbf{M} \dot{h}(t) \mathbf{x}(t-\boldsymbol{\tau}) \\
+\mathbf{M} h(t)\left(f_{1}(\widetilde{\mathbf{x}}(t)) \widehat{A}+f_{2}(\widetilde{\mathbf{x}}(t))\right)-\mathbf{K e}, \\
\dot{k}_{i}=\varepsilon_{i} e_{i}^{2}, \quad \varepsilon_{i}>0, i=1,2, \ldots, n, \\
\dot{\mathbf{e}}_{A}(t)=-f_{1}^{\mathrm{T}}(\widetilde{\mathbf{x}}(t)) h^{\mathrm{T}}(t) \mathbf{M e}(t), \\
\dot{\mathbf{e}}_{B}(t)=g_{1}(\mathbf{y}(t)) \mathbf{e}(t) .
\end{gathered}
$$

The proof is similar to Case 1 and is omitted here.

\section{Numerical Simulations}

In this section, four typical cases are provided to verify and show the effectiveness of the controller, parameter update rule, and control gain strength adapt law. The solver DDE23 in Matlab is used to integrate the delay differential equations.

4.1. Case 1: MFPLS between Two Five-Dimensional Hyperchaotic Systems with Identical Structures. In this subsection, we consider the case that $m=n$ and the drive and response system have the identical dimensions and structures. Recently, $\mathrm{Hu}$ [31] proposed a new five-dimensional hyperchaotic Lorenz system by introducing two state feedback controllers to the classical three-dimensional Lorenz system, which is described by

$$
\begin{gathered}
\dot{x}_{1}=-\sigma x_{1}+\sigma x_{2}+x_{4}, \\
\dot{x}_{2}=r x_{1}-x_{2}-x_{1} x_{3}-x_{5}, \\
\dot{x}_{3}=-\beta x_{3}+x_{1} x_{2}, \\
\dot{x}_{4}=-x_{1} x_{3}+d_{1} x_{4}, \\
\dot{x}_{5}=d_{2} x_{2},
\end{gathered}
$$

where $x_{1}, x_{2}, x_{3}, x_{4}$, and $x_{5}$ are state variables and $\sigma, \beta, r, d_{1}$, and $d_{2}$ are the unknown system parameters to be identified. When $\sigma=10, \beta=8 / 3, r=28, d_{1}=2$, and $d_{2} \in(2,12)$, the system is hyperchaotic with three positive LEs. $u_{1}, u_{2}, u_{3}, u_{4}$, and $u_{5}$ are the controllers to be designed.

The five-dimensional Lorenz hyperchaotic system, as the response system, is described as

$$
\begin{gathered}
\dot{y}_{1}=-\sigma y_{1}+\sigma y_{2}+y_{4}+u_{1}, \\
\dot{y}_{2}=r y_{1}-y_{2}-y_{1} y_{3}-y_{5}+u_{2}, \\
\dot{y}_{3}=-\beta y_{3}+y_{1} y_{2}+u_{3}, \\
\dot{y}_{4}=-y_{1} y_{3}+d_{1} y_{4}+u_{4}, \\
\dot{y}_{5}=d_{2} y_{2}+u_{5},
\end{gathered}
$$

where $y_{1}, y_{2}, y_{3}, y_{4}$, and $y_{5}$ are state variables, $\sigma, \beta, r, d_{1}$, and $d_{2}$ are the unknown system parameters to be identified. $u_{1}, u_{2}, u_{3}, u_{4}$, and $u_{5}$ are the controllers to be constructed so 
that the drive system (24) and the response system (25) can be synchronized in the sense of MFPLS.

We define the MFPLS error as follows:

$$
\begin{aligned}
& e_{1}(t)=y_{1}(t)-m_{1} h(t) x_{1}\left(t-\tau_{1}\right), \\
& e_{2}(t)=y_{2}(t)-m_{2} h(t) x_{2}\left(t-\tau_{2}\right), \\
& e_{3}(t)=y_{3}(t)-m_{3} h(t) x_{3}\left(t-\tau_{3}\right), \\
& e_{4}(t)=y_{4}(t)-m_{4} h(t) x_{4}\left(t-\tau_{4}\right), \\
& e_{5}(t)=y_{5}(t)-m_{5} h(t) x_{5}\left(t-\tau_{5}\right),
\end{aligned}
$$

where $m_{i}(i=1,2,3,4,5)$ is a scaling constant, $h(t)$ is a nonzero continuous differentiable function, and the time delay vector is $\boldsymbol{\tau}=\left(\tau_{1}, \tau_{2}, \tau_{3}, \tau_{4}, \tau_{5}\right)^{\mathrm{T}}$.

The time derivative of the error system (26) is as follows:

$$
\begin{aligned}
& \dot{e}_{1}(t)=\dot{y}_{1}(t)-m_{1} \dot{h}(t) x_{1}\left(t-\tau_{1}\right)-m_{1} h(t) \dot{x}_{1}\left(t-\tau_{1}\right), \\
& \dot{e}_{2}(t)=\dot{y}_{2}(t)-m_{2} \dot{h}(t) x_{2}\left(t-\tau_{2}\right)-m_{2} h(t) \dot{x}_{2}\left(t-\tau_{2}\right), \\
& \dot{e}_{3}(t)=\dot{y}_{3}(t)-m_{3} \dot{h}(t) x_{3}\left(t-\tau_{3}\right)-m_{3} h(t) \dot{x}_{3}\left(t-\tau_{3}\right), \\
& \dot{e}_{4}(t)=\dot{y}_{4}(t)-m_{4} \dot{h}(t) x_{4}\left(t-\tau_{4}\right)-m_{4} h(t) \dot{x}_{4}\left(t-\tau_{4}\right), \\
& \dot{e}_{5}(t)=\dot{y}_{5}(t)-m_{5} \dot{h}(t) x_{5}\left(t-\tau_{5}\right)-m_{5} h(t) \dot{x}_{5}\left(t-\tau_{5}\right) .
\end{aligned}
$$

Substituting (24) and (25) into (27), we can get the following:

$$
\begin{aligned}
\dot{e}_{1}(t)= & -\sigma y_{1}(t)+\sigma y_{2}(t)+y_{4}(t)+u_{1}(t) \\
& -m_{1} \dot{h}(t) x_{1}\left(t-\tau_{1}\right)-m_{1} h(t) \\
& \times\left(-\sigma x_{1}\left(t-\tau_{1}\right)+\sigma x_{2}\left(t-\tau_{1}\right)+x_{4}\left(t-\tau_{1}\right)\right), \\
\dot{e}_{2}(t)= & r y_{1}(t)-y_{2}(t)-y_{1}(t) y_{3}(t)-y_{5}(t)+u_{2}(t) \\
& -m_{2} \dot{h}(t) x_{2}\left(t-\tau_{2}\right)-m_{2} h(t) \\
& \times\left(r x_{1}\left(t-\tau_{2}\right)-x_{2}\left(t-\tau_{2}\right)\right. \\
& \left.-x_{1}\left(t-\tau_{2}\right) x_{3}\left(t-\tau_{2}\right)-x_{5}\left(t-\tau_{2}\right)\right), \\
\dot{e}_{3}(t)= & -\beta y_{3}(t)+y_{1}(t) y_{2}(t)+u_{3}(t) \\
& -m_{3} \dot{h}(t) x_{3}\left(t-\tau_{3}\right)-m_{3} h(t) \\
& \times\left(-\beta x_{3}\left(t-\tau_{3}\right)+x_{1}\left(t-\tau_{3}\right) x_{2}\left(t-\tau_{3}\right)\right), \\
\dot{e}_{4}(t)= & -y_{1}(t) y_{3}(t)+d_{1} y_{4}(t)+u_{4}(t) \\
& -m_{4} \dot{h}(t) x_{4}\left(t-\tau_{4}\right)-m_{4} h(t) \\
& \times\left(-x_{1}\left(t-\tau_{4}\right) x_{3}\left(t-\tau_{4}\right)+d_{1} x_{4}\left(t-\tau_{4}\right)\right), \\
\dot{e}_{5}(t)= & d_{2} y_{2}(t)+u_{5}(t)-m_{5} \dot{h}(t) x_{5}\left(t-\tau_{5}\right) \\
& -m_{5} h(t) d_{2} x_{2}\left(t-\tau_{5}\right) .
\end{aligned}
$$

Based on Theorem 3, the controller, parameter update rule, and control gain strength adapt law are described as follows:

$$
\begin{aligned}
& u_{1}(t)=\widehat{\sigma}\left(y_{1}(t)-y_{2}(t)\right)-y_{4}(t)+m_{1} \dot{h}(t) x_{1}\left(t-\tau_{1}\right) \\
& -m_{1} h(t)\left(\widehat{\sigma} x_{1}\left(t-\tau_{1}\right)-\widehat{\sigma} x_{2}\left(t-\tau_{1}\right)-x_{4}\left(t-\tau_{1}\right)\right) \\
& -k_{1} e_{1} \\
& u_{2}(t)=-\widehat{r} y_{1}(t)+y_{2}(t)+y_{1}(t) y_{3}(t)+y_{5}(t) \\
& +m_{2} \dot{h}(t) x_{2}\left(t-\tau_{2}\right)-m_{2} h(t) \\
& \times\left(-\widehat{r} x_{1}\left(t-\tau_{2}\right)+x_{2}\left(t-\tau_{2}\right)+x_{1}\left(t-\tau_{2}\right)\right. \\
& \left.\times x_{3}\left(t-\tau_{2}\right)+x_{5}\left(t-\tau_{2}\right)\right)-k_{2} e_{2}, \\
& u_{3}(t)=\widehat{\beta} y_{3}(t)-y_{1}(t) y_{2}(t)+m_{3} \dot{h}(t) x_{3}\left(t-\tau_{3}\right) \\
& -m_{3} h(t)\left(\widehat{\beta} x_{3}\left(t-\tau_{3}\right)-x_{1}\left(t-\tau_{3}\right) x_{2}\left(t-\tau_{3}\right)\right) \\
& -k_{3} e_{3} \\
& u_{4}(t)=y_{1}(t) y_{3}(t)-\widehat{d}_{1} y_{4}(t)+m_{4} \dot{h}(t) x_{4}\left(t-\tau_{4}\right) \\
& -m_{4} h(t)\left(x_{1}\left(t-\tau_{4}\right) x_{3}\left(t-\tau_{4}\right)-\widehat{d}_{1} x_{4}\left(t-\tau_{4}\right)\right) \\
& -k_{4} e_{4} \\
& u_{5}(t)=-\widehat{d}_{2} y_{2}(t)+m_{5} \dot{h}(t) x_{5}\left(t-\tau_{5}\right) \\
& +m_{5} h(t) \widehat{d}_{2} x_{2}\left(t-\tau_{5}\right)-k_{5} e_{5},
\end{aligned}
$$

$$
\begin{aligned}
\dot{e}_{\sigma}=\dot{\hat{\sigma}}= & -e_{1}\left(y_{1}(t)-y_{2}(t)\right) \\
& +m_{1} h(t) e_{1}\left(x_{1}\left(t-\tau_{1}\right)-x_{2}\left(t-\tau_{1}\right)\right), \\
\dot{e}_{\beta}= & \dot{\hat{\beta}}=-e_{3} y_{3}(t)+m_{3} h(t) e_{3} x_{3}\left(t-\tau_{3}\right), \\
\dot{e}_{r}= & \dot{\hat{r}}=e_{2} y_{1}(t)-m_{2} h(t) e_{2} x_{1}\left(t-\tau_{2}\right), \\
\dot{e}_{d_{1}}= & \dot{\vec{d}}_{1}=e_{4} y_{4}(t)-m_{4} h(t) e_{4} x_{4}\left(t-\tau_{4}\right), \\
\dot{e}_{d_{2}}= & \dot{\vec{d}}_{2}=e_{5} y_{2}(t)-m_{5} h(t) e_{5} x_{2}\left(t-\tau_{5}\right), \\
& \dot{k}_{i}=\varepsilon_{i} e_{i}^{2}, \quad \varepsilon_{i}>0, \quad i=1,2, \ldots, 5,
\end{aligned}
$$

where $\widehat{\sigma}, \widehat{\beta}, \widehat{r}, \widehat{d}_{1}$, and $\widehat{d}_{2}$ are the estimation of uncertain parameters $\sigma, \beta, r, d_{1}$, and $d_{2}$, respectively, $e_{\sigma}=\widehat{\sigma}-\sigma, e_{\beta}=$ $\widehat{\beta}-\beta, e_{r}=\widehat{r}-r, e_{d_{1}}=\widehat{d}_{1}-d_{1}$, and $e_{d_{2}}=\widehat{d}_{2}-d_{2}$ are the corresponding parameter errors.

Proof. The Lyapunov function is chosen as

$$
\begin{aligned}
V(t)=\frac{1}{2}\left(e_{1}^{\mathrm{T}}(t) e_{1}(t)+e_{2}^{\mathrm{T}}(t) e_{2}(t)+e_{3}^{\mathrm{T}}(t) e_{3}(t)\right. \\
\\
\quad+e_{4}^{\mathrm{T}}(t) e_{4}(t)+e_{5}^{\mathrm{T}}(t) e_{5}(t)+e_{\sigma}^{\mathrm{T}}(t) e_{\sigma}(t)
\end{aligned}
$$




$$
\begin{aligned}
& +e_{\beta}^{\mathrm{T}}(t) e_{\beta}(t)+e_{r}^{\mathrm{T}}(t) e_{r}(t)+e_{d_{1}}^{\mathrm{T}}(t) e_{d_{1}}(t) \\
& \left.+e_{d_{2}}^{\mathrm{T}}(t) e_{d_{2}}(t)\right)+\frac{1}{2} \sum_{i=1}^{5} \frac{1}{\varepsilon_{i}}\left(k_{i}-k\right)^{2}
\end{aligned}
$$

where $k>0$ is a positive constant.

Then the time derivation of the Lyapunov function along the trajectory of error systems (27) is as follows:

$$
\begin{aligned}
\dot{V}(t)= & e_{1}^{\mathrm{T}}(t) \dot{e}_{1}(t)+e_{2}^{\mathrm{T}}(t) \dot{e}_{2}(t)+e_{3}^{\mathrm{T}}(t) \dot{e}_{3}(t) \\
& +e_{4}^{\mathrm{T}}(t) \dot{e}_{4}(t)+e_{5}^{\mathrm{T}}(t) \dot{e}_{5}(t)+e_{\sigma}^{\mathrm{T}}(t) \dot{e}_{\sigma}(t) \\
& +e_{\beta}^{\mathrm{T}}(t) \dot{e}_{\beta}(t)+e_{r}^{\mathrm{T}}(t) \dot{e}_{r}(t)+e_{d_{1}}^{\mathrm{T}}(t) \dot{e}_{d_{1}}(t) \\
& +e_{d_{2}}^{\mathrm{T}}(t) \dot{e}_{d_{2}}(t)+\dot{k}_{i} \sum_{i=1}^{5} \frac{1}{\varepsilon_{i}}\left(k_{i}-k\right)^{2} .
\end{aligned}
$$

Constituting (28), (29), (30), and (31) into (33), we get the following:

$$
\begin{aligned}
\dot{V}(t) & =-k\left(e_{1}^{\mathrm{T}}(t) e_{1}+e_{2}^{\mathrm{T}}(t) e_{2}+e_{3}^{\mathrm{T}}(t) e_{3}+e_{4}^{\mathrm{T}}(t) e_{4}+e_{5}^{\mathrm{T}}(t) e_{5}\right) \\
& <0
\end{aligned}
$$

$V(t)$ is positive definite and $\dot{V}(t)<0$; thus, according to Barbalat's Lemma, MFPLS between the drive system (24) and the response system (25) is achieved. The uncertain parameters and control gain strengths can be identified and defined as well.

To verify the effectiveness of the proposed synchronization method, the numerical simulation is performed. The system parameters are chosen as $\sigma=10, \beta=8 / 3, r=28, d_{1}=2$, and $d_{2}=8$, such that the drive system (24) and the response system (25) can exhibit hyperchaotic behaviors without control. The initial conditions of the drive system (24) and the response system $(25)$ are taken as $\mathbf{x}(0)=(-2,1,4,2,-3)^{\mathrm{T}}$, $\mathbf{y}(0)=(-2,-2,3,4,1)^{\mathrm{T}}$, respectively. The initial values of the control gain strengths are selected as $k_{1}(0)=k_{2}(0)=$ $k_{3}(0)=k_{4}(0)=k_{5}(0)=6$, and the constants are $\varepsilon_{1}=$ $\varepsilon_{2}=\varepsilon_{3}=\varepsilon_{4}=\varepsilon_{5}=10$. The initial values of the unknown parameters are set as $\widehat{\sigma}(0)=\widehat{\beta}(0)=\widehat{r}(0)=\widehat{d}_{1}(0)=\widehat{d}_{2}(0)=$ 0.001 . The time delays are arbitrarily chosen as $\left(\tau_{1}, \tau_{2}, \tau_{3}, \tau_{4}\right.$, $\left.\tau_{5}\right)=(0.2,0.1,0.3,0.1,0.4)$. The scaling constants are randomly taken as $\left(m_{1}, m_{2}, m_{3}, m_{4}, m_{5}\right)=(2,-3,-2,1,-4)$, and nonzero continuous differentiable function is selected as $h(t)=2 \sin (t)+1$.

The corresponding simulation results are illustrated in Figures 1, 2, and 3. Figure 1 displays that the synchronization error variables $e_{1}, e_{2}, e_{3}, e_{4}$, and $e_{5}$ converge to zero after a transient time, respectively. Figure 2 shows that the estimated values of the uncertain parameters approach to the true values; that is, $\widehat{\sigma} \rightarrow 10, \widehat{\beta} \rightarrow 8 / 3, \widehat{r} \rightarrow 28, \widehat{d}_{1} \rightarrow 2$, and $\widehat{d}_{2} \rightarrow 8$ as $t \rightarrow \infty$. Figure 3 depicts the control gain strengths adapt themselves to a certain value, that is, $k_{1}=$ $32.34, k_{2}=88.97, k_{3}=10.82, k_{4}=10.77$, and $k_{5}=27.67$ as $t \rightarrow \infty$. In Figure 3, the upper right small figure is

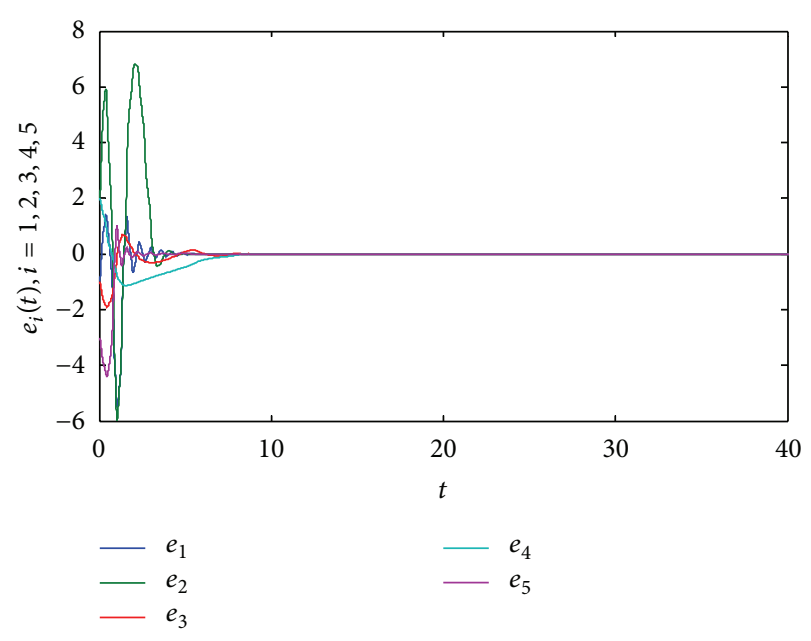

FIGURE 1: Time evolution of MFPLS errors between systems (24) and (25).

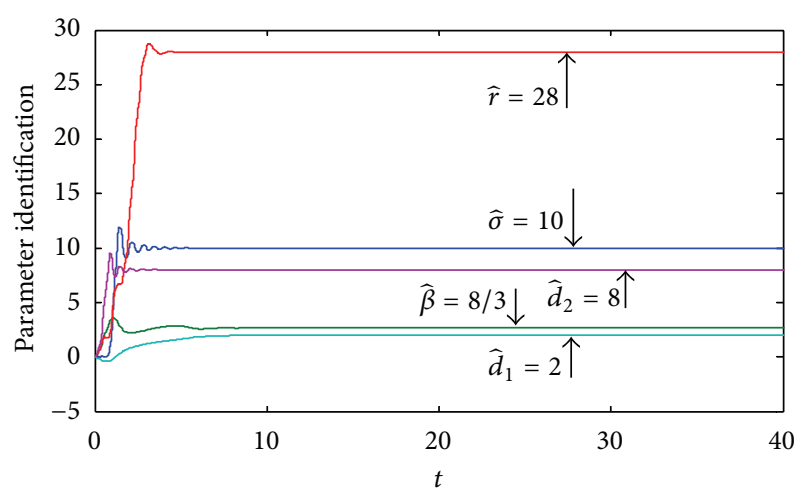

FIGURE 2: Time evolution of parameter estimation for the systems (24) and (25).

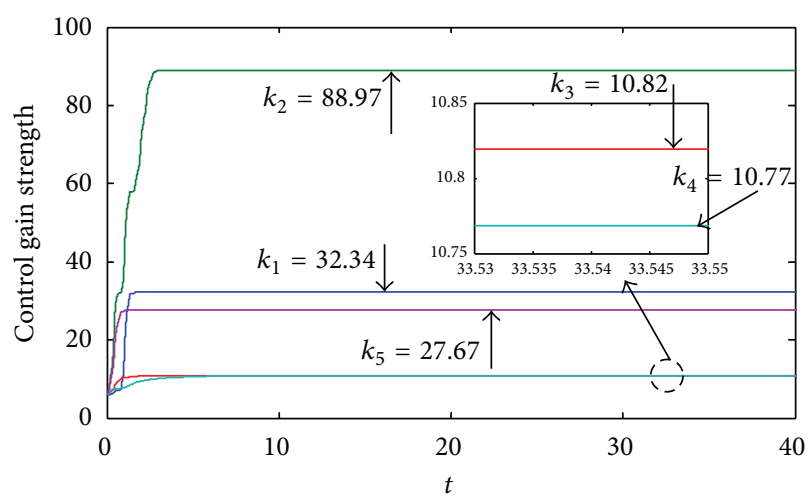

FIgURE 3: Time evolution of the control gain strength.

the magnified drawing of the circling part. These results show that the MFPLS has been achieved with the adaptive controller (29) and the parameter update rule (30), and control gain strengths can be identified automatically.

4.2. Case 2: MFPLS between Two Four-Dimensional Hyperchaotic Systems with Different Structures. In this subsection, 
we consider the case that $m=n$ and the drive and response system have the same dimensions and different structures. Furthermore, we select the four-dimensional Lü hyperchaotic system [32] and the four-dimensional Lorenz-Stenflo (LS) hyperchaotic system [33] as the drive system and the response system, respectively.

The four-dimensional Lü hyperchaotic system [32] is given by the following equations:

$$
\begin{gathered}
\dot{x}_{1}=a_{1}\left(x_{2}-x_{1}\right)+x_{4}, \\
\dot{x}_{2}=-x_{1} x_{3}+c_{1} x_{2}, \\
\dot{x}_{3}=x_{1} x_{2}-b_{1} x_{3}, \\
\dot{x}_{4}=x_{1} x_{3}+d_{1} x_{4},
\end{gathered}
$$

where $x_{1}, x_{2}, x_{3}$, and $x_{4}$ are state variables and $a_{1}, b_{1}, c_{1}$, and $d_{1}$ are uncertain system parameters. When $a_{1}=36, b_{1}=3$, $c_{1}=20$, and $d_{1}=1$, system (35) is hyperchaotic.

Lorenz-Stenflo (LS) hyperchaotic system [33] is described as

$$
\begin{gathered}
\dot{y}_{1}=\alpha\left(y_{2}-y_{1}\right)+\beta y_{4}+u_{1}, \\
\dot{y}_{2}=\gamma y_{1}-y_{1} y_{3}-y_{2}+u_{2}, \\
\dot{y}_{3}=y_{1} y_{2}-\theta y_{3}+u_{3}, \\
\dot{y}_{4}=-y_{1}-\alpha y_{4}+u_{4},
\end{gathered}
$$

where $y_{1}, y_{2}, y_{3}$, and $y_{4}$ are state variables, $\alpha, \beta, \gamma$, and $\theta$ are the unknown system parameters. When $\alpha=1, \beta=1.5, \gamma=26$, and $\theta=0.7$, system (36) exhibits hyperchaotic behavior. And $u_{1}, u_{2}, u_{3}$, and $u_{4}$ are the controllers such that the two hyperchaotic systems can be synchronized in the sense of MFPLS.

We define the error as

$$
\begin{aligned}
& e_{1}(t)=y_{1}(t)-m_{1} h(t) x_{1}\left(t-\tau_{1}\right), \\
& e_{2}(t)=y_{2}(t)-m_{2} h(t) x_{2}\left(t-\tau_{2}\right), \\
& e_{3}(t)=y_{3}(t)-m_{3} h(t) x_{3}\left(t-\tau_{3}\right), \\
& e_{4}(t)=y_{4}(t)-m_{4} h(t) x_{4}\left(t-\tau_{4}\right),
\end{aligned}
$$

where $m_{i}(i=1,2,3,4)$ is a scaling constant, $h(t)$ is a nonzero continuous differentiable function, and $\tau=\left(\tau_{1}, \tau_{2}, \tau_{3}, \tau_{4}\right)^{\mathrm{T}}$ are the time delays.

The time derivative of the error system (37) is

$$
\begin{aligned}
& \dot{e}_{1}(t)=\dot{y}_{1}(t)-m_{1} \dot{h}(t) x_{1}\left(t-\tau_{1}\right)-m_{1} h(t) \dot{x}_{1}\left(t-\tau_{1}\right), \\
& \dot{e}_{2}(t)=\dot{y}_{2}(t)-m_{2} \dot{h}(t) x_{2}\left(t-\tau_{2}\right)-m_{2} h(t) \dot{x}_{2}\left(t-\tau_{2}\right), \\
& \dot{e}_{3}(t)=\dot{y}_{3}(t)-m_{3} \dot{h}(t) x_{3}\left(t-\tau_{3}\right)-m_{3} h(t) \dot{x}_{3}\left(t-\tau_{3}\right), \\
& \dot{e}_{4}(t)=\dot{y}_{4}(t)-m_{4} \dot{h}(t) x_{4}\left(t-\tau_{4}\right)-m_{4} h(t) \dot{x}_{4}\left(t-\tau_{4}\right) .
\end{aligned}
$$

Substituting (35) and (36) into (38), the error dynamical system is obtained as follows:

$$
\begin{aligned}
\dot{e}_{1}(t)= & \alpha\left(y_{2}(t)-y_{1}(t)\right)+\beta y_{4}(t)+u_{1}(t) \\
& -m_{1} \dot{h}(t) x_{1}\left(t-\tau_{1}\right)-m_{1} h(t) \\
& \times\left(a_{1}\left(x_{2}\left(t-\tau_{1}\right)-x_{1}\left(t-\tau_{1}\right)\right)+x_{4}\left(t-\tau_{1}\right)\right) \\
& -k_{1} e_{1} \\
\dot{e}_{2}(t)= & \gamma y_{1}(t)-y_{1}(t) y_{3}(t)-y_{2}(t)+u_{2}(t) \\
& -m_{2} \dot{h}(t) x_{2}\left(t-\tau_{2}\right)-m_{2} h(t) \\
& \times\left(-x_{1}\left(t-\tau_{2}\right) x_{3}\left(t-\tau_{2}\right)+c_{1} x_{2}\left(t-\tau_{2}\right)\right)-k_{2} e_{2}, \\
\dot{e}_{3}(t)= & y_{1}(t) y_{2}(t)-\theta y_{3}(t)+u_{3}(t) \\
& -m_{3} \dot{h}(t) x_{3}\left(t-\tau_{3}\right)-m_{3} h(t) \\
& \times\left(x_{1}\left(t-\tau_{3}\right) x_{2}\left(t-\tau_{3}\right)-b_{1} x_{3}\left(t-\tau_{3}\right)\right)-k_{3} e_{3}, \\
\dot{e}_{4}(t)= & -y_{1}(t)-\alpha y_{4}(t)+u_{4}(t) \\
& -m_{4} \dot{h}(t) x_{4}\left(t-\tau_{4}\right)-m_{4} h(t) \\
& \times\left(x_{1}\left(t-\tau_{4}\right) x_{3}\left(t-\tau_{4}\right)+d_{1} x_{4}\left(t-\tau_{4}\right)\right)-k_{4} e_{4} .
\end{aligned}
$$

Based on Theorem 3, the controller, parameter update rule, and control gain strength adapt law are constructed as follows:

$$
\begin{aligned}
& u_{1}(t)=-\widehat{\alpha}\left(y_{2}(t)-y_{1}(t)\right)-\widehat{\beta} y_{4}(t) \\
&+m_{1} \dot{h}(t) x_{1}\left(t-\tau_{1}\right)-m_{1} h(t) \\
& \times\left(-\widehat{a}_{1}\left(x_{2}\left(t-\tau_{1}\right)-x_{1}\left(t-\tau_{1}\right)\right)-x_{4}\left(t-\tau_{1}\right)\right) \\
&-k_{1} e_{1}, \\
& u_{2}(t)=-\widehat{\gamma} y_{1}(t)+y_{1}(t) y_{3}(t)+y_{2}(t) \\
&+m_{2} \dot{h}(t) x_{2}\left(t-\tau_{2}\right)-m_{2} h(t) \\
& \times\left(x_{1}\left(t-\tau_{2}\right) x_{3}\left(t-\tau_{2}\right)-\widehat{c}_{1} x_{2}\left(t-\tau_{2}\right)\right) \\
&-k_{2} e_{2}, \\
& u_{3}(t)=- y_{1}(t) y_{2}(t)+\widehat{\theta} y_{3}(t)+m_{3} \dot{h}(t) x_{3}\left(t-\tau_{3}\right) \\
&- m_{3} h(t)\left(-x_{1}\left(t-\tau_{3}\right) x_{2}\left(t-\tau_{3}\right)+\widehat{b}_{1} x_{3}\left(t-\tau_{3}\right)\right) \\
&- k_{3} e_{3}, \\
& u_{4}(t)=y_{1}(t)+\widehat{\alpha} y_{4}(t)+m_{4} \dot{h}(t) x_{4}\left(t-\tau_{4}\right) \\
&-m_{4} h(t)\left(-x_{1}\left(t-\tau_{4}\right) x_{3}\left(t-\tau_{4}\right)-\widehat{d}_{1} x_{4}\left(t-\tau_{4}\right)\right) \\
&-k_{4} e_{4},
\end{aligned}
$$




$$
\begin{gathered}
\dot{e}_{a_{1}}=\dot{\hat{a}}_{1}=-m_{1} h(t) e_{1}\left(x_{2}\left(t-\tau_{1}\right)-x_{1}\left(t-\tau_{1}\right)\right), \\
\dot{e}_{b_{1}}=\dot{\hat{b}}_{1}=m_{3} h(t) e_{3} x_{3}\left(t-\tau_{3}\right), \\
\dot{e}_{c_{1}}=\dot{\widehat{c}}_{1}=-m_{2} h(t) e_{2} x_{2}\left(t-\tau_{2}\right), \\
\dot{e}_{d_{1}}=\dot{\hat{d}}_{1}=-m_{4} h(t) e_{4} x_{4}\left(t-\tau_{4}\right), \\
\dot{e}_{\alpha}=\dot{\hat{\alpha}}=-e_{4} y_{4}(t)+e_{1}\left(y_{2}(t)-y_{1}(t)\right) \\
\dot{e}_{\beta}=\dot{\hat{\beta}}=e_{1} y_{4}(t), \\
\dot{e}_{\gamma}=\dot{\hat{\gamma}}=e_{2} y_{1}(t) \\
\dot{e}_{\theta}=\dot{\hat{\theta}}=-e_{3} y_{3}(t), \\
\dot{k}_{i}=\varepsilon_{i} e_{i}^{2}, \quad \varepsilon_{i}>0, i=1,2, \ldots, 4
\end{gathered}
$$

where $\widehat{a}_{1}, \widehat{b}_{1}, \widehat{c}_{1}, \widehat{d}_{1}, \widehat{\alpha}, \widehat{\beta}, \widehat{\gamma}$, and $\widehat{\theta}$ are estimated values of the unknown parameters $a_{1}, b_{1}, c_{1}, d_{1}, \alpha, \beta, \gamma$, and $\theta$, respectively, and $e_{a_{1}}=\widehat{a}_{1}-a_{1}, e_{b_{1}}=\widehat{b}_{1}-b_{1}, e_{c_{1}}=\widehat{c}_{1}-c_{1}, e_{d_{1}}=\widehat{d}_{1}-d_{1}$, $e_{\alpha}=\widehat{\alpha}-\alpha, e_{\beta}=\widehat{\beta}-\beta, e_{\gamma}=\widehat{\gamma}-\gamma$, and $e_{\theta}=\widehat{\theta}-\theta$ are the parameter errors.

Proof. The Lyapunov function is chosen as

$$
\begin{aligned}
V(t)=\frac{1}{2}\left(e_{1}^{\mathrm{T}}(t) e_{1}(t)+e_{2}^{\mathrm{T}}(t) e_{2}(t)+e_{3}^{\mathrm{T}}(t) e_{3}(t)\right. \\
+e_{4}^{\mathrm{T}}(t) e_{4}(t)+e_{a_{1}}^{\mathrm{T}}(t) e_{a_{1}}(t)+e_{b_{1}}^{\mathrm{T}}(t) e_{b_{1}}(t) \\
+e_{c_{1}}^{\mathrm{T}}(t) e_{c_{1}}(t)+e_{d_{1}}^{\mathrm{T}}(t) e_{d_{1}}(t)+e_{\alpha}^{\mathrm{T}}(t) e_{\alpha}(t) \\
\left.+e_{\beta}^{\mathrm{T}}(t) e_{\beta}(t)+e_{\gamma}^{\mathrm{T}}(t) e_{\gamma}(t)+e_{\theta}^{\mathrm{T}}(t) e_{\theta}(t)\right) \\
+\frac{1}{2} \sum_{i=1}^{4} \frac{1}{\varepsilon_{i}}\left(k_{i}-k\right)^{2},
\end{aligned}
$$

where $k>0$ is a positive constant.

Then the time derivation of the Lyapunov function along the trajectory of error systems (39) is

$$
\begin{aligned}
\dot{V}(t)= & e_{1}^{\mathrm{T}}(t) \dot{e}_{1}(t)+e_{2}^{\mathrm{T}}(t) \dot{e}_{2}(t)+e_{3}^{\mathrm{T}}(t) \dot{e}_{3}(t) \\
& +e_{4}^{\mathrm{T}}(t) \dot{e}_{4}(t)+e_{a_{1}}^{\mathrm{T}}(t) \dot{e}_{a_{1}}(t)+e_{b_{1}}^{\mathrm{T}}(t) \dot{e}_{b_{1}}(t) \\
& +e_{c_{1}}^{\mathrm{T}}(t) \dot{e}_{c_{1}}(t)+e_{d_{1}}^{\mathrm{T}}(t) \dot{e}_{d_{1}}(t)+e_{\alpha}^{\mathrm{T}}(t) \dot{e}_{\alpha}(t) \\
& +e_{\beta}^{\mathrm{T}}(t) \dot{e}_{\beta}(t)+e_{\gamma}^{\mathrm{T}}(t) \dot{e}_{\gamma}(t)+e_{\theta}^{\mathrm{T}}(t) \dot{e}_{\theta}(t) \\
& +\dot{k}_{i} \sum_{i=1}^{4} \frac{1}{\varepsilon_{i}}\left(k_{i}-k\right)^{2} .
\end{aligned}
$$

Constituting (39), (40), (41), and (42) into (44), we get the following:

$$
\dot{V}(t)=-k\left(e_{1}^{\mathrm{T}}(t) e_{1}+e_{2}^{\mathrm{T}}(t) e_{2}+e_{3}^{\mathrm{T}}(t) e_{3}+e_{4}^{\mathrm{T}}(t) e_{4}\right)<0 .
$$

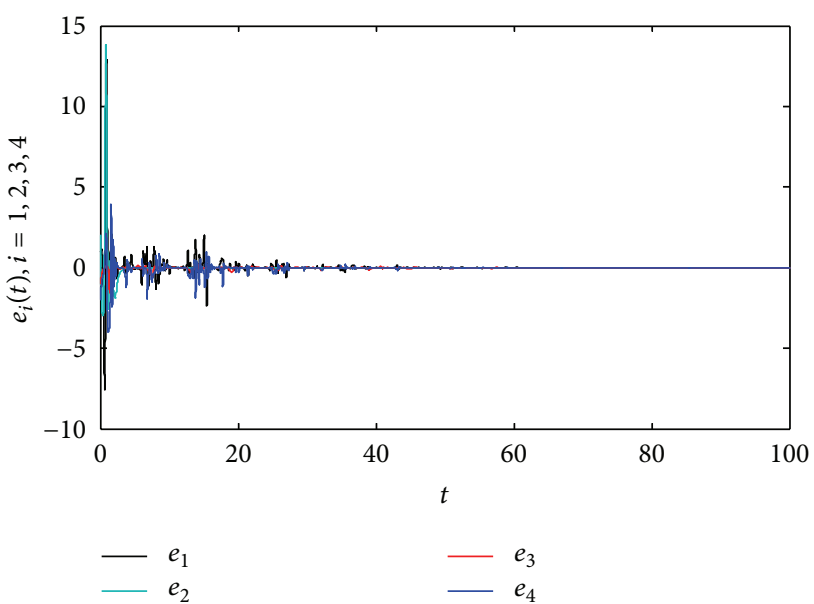

FIGURE 4: Time evolution of MFPLS errors between systems (35) and (36).

$V(t)$ is positive definite and $\dot{V}(t)<0$; thus, according to Barbalat's Lemma, MFPLS between the drive system (35) and the response system (36) is achieved. The uncertain parameters and control gain strengths can be identified and defined as well.

In the numerical simulation, the system parameters are selected as $a_{1}=36, b_{1}=3, c_{1}=20, d_{1}=1, \alpha=1, \beta=1.5$, $\gamma=26$, and $\theta=0.7$, such that the drive system (35) and the response system (36) are hyperchaotic with no control applied. The initial conditions of the drive system (35) and the response system (36) are taken as $\mathbf{x}(0)=(-2,-1,-4,2)^{\mathrm{T}}$, $\mathbf{y}(0)=(-2,-1,3,5)^{\mathrm{T}}$, respectively. The initial values of the unknown parameters are arbitrarily set as $\widehat{a}_{1}(0)=\widehat{b}_{1}(0)=$ $\widehat{c}_{1}(0)=\widehat{d}_{1}(0)=\widehat{\alpha}(0)=\widehat{\beta}(0)=\widehat{\gamma}(0)=\widehat{\theta}(0)=0.001$. The constants are $\varepsilon_{1}=\varepsilon_{2}=\varepsilon_{3}=\varepsilon_{4}=5$ and the initial values of the control gain strength are chosen as $k_{1}(0)=$ $k_{2}(0)=k_{3}(0)=k_{4}(0)=10$. The scaling constants are set as $\left(m_{1}, m_{2}, m_{3}, m_{4}\right)=(-3,2,-1,2)$, and nonzero continuous differentiable function is selected as $h(t)=\sin (t)$. The time delays are chosen as $\left(\tau_{1}, \tau_{2}, \tau_{3}, \tau_{4}\right)=(0.1,0.2,0.3,0.4)$.

Numerical results are displayed in Figures $4-7$. Figure 4 shows the time evolution of the MFPLS errors, which displays that the errors tend to zero as $t \rightarrow \infty$. Figures 5 and 6 depict the evolution of the estimated parameters of Lü hyperchaotic system and Lorenz-Stenflo (LS) hyperchaotic system, which displays that the estimates of the uncertain parameters converge to $a_{1}=36, b_{1}=3, c_{1}=20, d_{1}=1, \alpha=1, \beta=1.5$, $\gamma=26$, and $\theta=0.7$ as $t \rightarrow \infty$, respectively. Figure 7 illustrates that the control gain strengths approach to some certain values, that is, $k_{1}=73.95, k_{2}=74.16, k_{3}=12.34$, and $k_{4}=$ 35.46 as $t \rightarrow \infty$. In Figure 7 , the middle small figure is the magnified drawing of the circling part. These results show that MFPLS between four-dimensional Lü hyperchaotic system and four-dimensional Lorenz-Stenflo (LS) hyperchaotic system is realized by using the adaptive controllers (40) and the parameter update rule (41), and the control gain strength can be gotten adaptively.

4.3. Case 3: MFPLS between a Four-Dimensional Hyperchaotic System and a Three-Dimensional Chaotic System. In this 


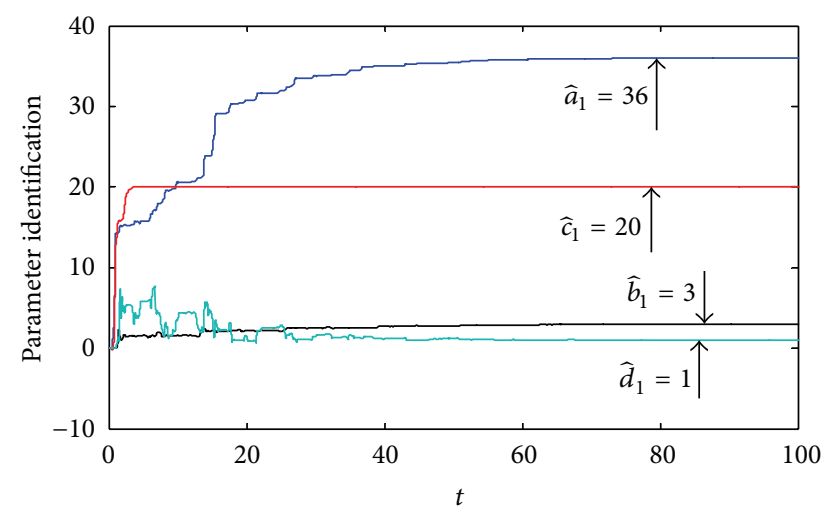

Figure 5: Time evolution of parameter estimation for Lü hyperchaotic system (35).

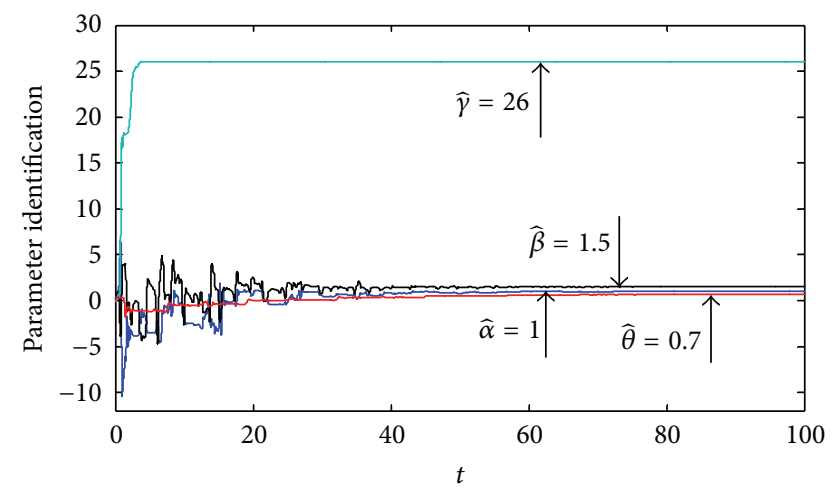

FIgURE 6: Time evolution of parameter estimation for LorenzStenflo (LS) hyperchaotic system (36).

subsection, we consider the case that $m>n$ and the dimension of drive system is larger than that of the response system.

The four-dimensional Lorenz-Stenflo (LS) hyperchaotic system [33], as the drive system, is given by

$$
\begin{gathered}
\dot{x}_{1}=\alpha\left(x_{2}-x_{1}\right)+\beta x_{4}, \\
\dot{x}_{2}=\gamma x_{1}-x_{1} x_{3}-x_{2}, \\
\dot{x}_{3}=x_{1} x_{2}-\theta x_{3}, \\
\dot{x}_{4}=-x_{1}-\alpha x_{4},
\end{gathered}
$$

where $x_{1}, x_{2}, x_{3}$, and $x_{4}$ are state variables and $\alpha, \beta, \gamma$, and $\theta$ are the uncertain system parameters to be estimated. When $\alpha=1, \beta=1.5, \gamma=26$, and $\theta=0.7$, system (46) exhibits hyperchaotic behavior.

In order to achieve the full-state MFPLS, an auxiliary state should be added to the response system. Since adding subcontroller to the response system to compensate it as extradimensions is a practicable way [21], the auxiliary state is $\dot{y}_{4}=$ $u_{4}$.

Then, the three-dimensional Lorenz system, as the response system, is described by

$$
\begin{gathered}
\dot{y}_{1}=l\left(y_{2}-y_{1}\right)+u_{1}, \\
\dot{y}_{2}=m y_{1}-y_{2}-y_{1} y_{3}+u_{2}, \\
\dot{y}_{3}=-n y_{3}+y_{1} y_{2}+u_{3}, \\
\dot{y}_{4}=u_{4},
\end{gathered}
$$

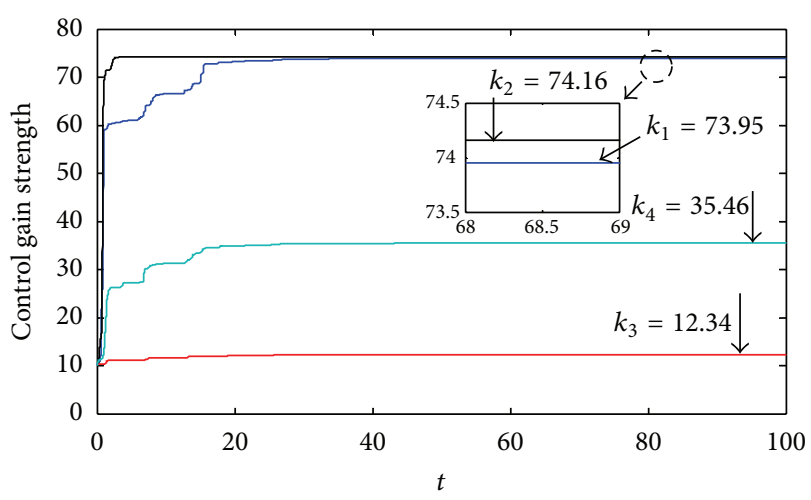

FIGURE 7: Time evolution of the control gain strength.

where $y_{1}, y_{2}, y_{3}$, and $y_{4}$ are state variables $l, m$, and $n$ are the unknown system parameters. When $l=10, m=28, n=8 / 3$, system (47) is chaotic, $u_{1}, u_{2}, u_{3}$, and $u_{4}$ are the controllers to be designed.

The MFPLS error is defined as

$$
\begin{aligned}
& e_{1}(t)=y_{1}(t)-m_{1} h(t) x_{1}\left(t-\tau_{1}\right), \\
& e_{2}(t)=y_{2}(t)-m_{2} h(t) x_{2}\left(t-\tau_{2}\right), \\
& e_{3}(t)=y_{3}(t)-m_{3} h(t) x_{3}\left(t-\tau_{3}\right), \\
& e_{4}(t)=y_{4}(t)-m_{4} h(t) x_{4}\left(t-\tau_{4}\right),
\end{aligned}
$$

where $m_{i}(i=1,2,3,4)$ is a scaling constant, $h(t)$ is a nonzero continuous differentiable function, and $\boldsymbol{\tau}=\left(\tau_{1}, \tau_{2}, \tau_{3}, \tau_{4}\right)^{\mathrm{T}}$ are the time delays.

Based on Theorem 3, the controller, parameter update rules, and control gain strength adapt laws are as follows:

$$
\begin{aligned}
u_{1}(t)= & -\widehat{l}\left(y_{2}(t)-y_{1}(t)\right)+m_{1} \dot{h}(t) x_{1}\left(t-\tau_{1}\right)-m_{1} h(t) \\
& \times\left(-\widehat{\alpha}\left(x_{2}\left(t-\tau_{1}\right)-x_{1}\left(t-\tau_{1}\right)\right)-\widehat{\beta} x_{4}\left(t-\tau_{1}\right)\right) \\
& -k_{1} e_{1}, \\
u_{2}(t)=- & \widehat{m} y_{1}+y_{2}+y_{1} y_{3}+m_{2} \dot{h}(t) x_{2}\left(t-\tau_{2}\right)-m_{2} h(t) \\
\times & \left(-\widehat{\gamma} x_{1}\left(t-\tau_{2}\right)+x_{1}\left(t-\tau_{2}\right) x_{3}\left(t-\tau_{2}\right)+x_{2}\left(t-\tau_{2}\right)\right) \\
- & k_{2} e_{2}, \\
u_{3}(t)= & +\widehat{n} y_{3}-y_{1} y_{2}+m_{3} \dot{h}(t) x_{3}\left(t-\tau_{3}\right)-m_{3} h(t) \\
\times & \left(-x_{1}\left(t-\tau_{3}\right) x_{2}\left(t-\tau_{3}\right)+\widehat{\theta} x_{3}\left(t-\tau_{3}\right)\right)-k_{3} e_{3}, \\
u_{4}(t)= & +m_{4} \dot{h}(t) x_{4}\left(t-\tau_{4}\right) \\
& -m_{4} h(t)\left(x_{1}\left(t-\tau_{4}\right)+\widehat{\alpha} x_{4}\left(t-\tau_{4}\right)\right)-k_{4} e_{4},
\end{aligned}
$$

$$
\begin{aligned}
& \dot{e}_{\alpha}=\dot{\hat{\alpha}}=-m_{1} h(t) e_{1}\left(x_{2}\left(t-\tau_{1}\right)-x_{1}\left(t-\tau_{1}\right)\right) \\
&+m_{4} h(t) e_{4} x_{4}\left(t-\tau_{4}\right), \\
& \dot{e}_{\beta}=\dot{\hat{\beta}}=-m_{1} h(t) e_{1} x_{4}\left(t-\tau_{1}\right),
\end{aligned}
$$




$$
\begin{gathered}
\dot{e}_{\gamma}=\dot{\hat{\gamma}}=-m_{2} h(t) e_{2} x_{1}\left(t-\tau_{2}\right), \\
\dot{e}_{\theta}=\dot{\hat{\theta}}=m_{3} h(t) e_{3} x_{3}\left(t-\tau_{3}\right), \\
\dot{e}_{l}=\dot{\hat{l}}=\left(y_{2}(t)-y_{1}(t)\right) e_{1}, \\
\dot{e}_{m}=\dot{\widehat{\hat{m}}}=e_{2} y_{1}(t), \\
\dot{e}_{n}=\dot{\hat{n}}=-e_{3} y_{3}(t), \\
\dot{k}_{i}=\varepsilon_{i} e_{i}^{2}, \quad \varepsilon_{i}>0, \quad i=1,2, \ldots, 4,
\end{gathered}
$$

where $\widehat{\alpha}, \widehat{\beta}, \widehat{\gamma}, \widehat{\theta}, \widehat{l}, \widehat{m}$, and $\widehat{n}$ are estimated values of the unknown parameters $\alpha, \beta, \gamma, \theta, l, m$, and $n$, respectively and $e_{\alpha}=\widehat{\alpha}-\alpha, e_{\beta}=\widehat{\beta}-\beta, e_{\gamma}=\widehat{\gamma}-\gamma, e_{\theta}=\widehat{\theta}-\theta, e_{l}=\widehat{l}-l$, $e_{m}=\widehat{m}-m$, and $e_{n}=\widehat{n}-n$ are the parameter errors.

Proof. The Lyapunov function is chosen as

$$
\begin{aligned}
V(t)=\frac{1}{2}\left(e_{1}^{\mathrm{T}}(t) e_{1}(t)+e_{2}^{\mathrm{T}}(t) e_{2}(t)+e_{3}^{\mathrm{T}}(t) e_{3}(t)\right. \\
+e_{4}^{\mathrm{T}}(t) e_{4}(t)+e_{\alpha}^{\mathrm{T}}(t) e_{\alpha}(t)+e_{\beta}^{\mathrm{T}}(t) e_{\beta}(t) \\
+e_{\gamma}^{\mathrm{T}}(t) e_{\gamma}(t)+e_{\theta}^{\mathrm{T}}(t) e_{\theta}(t)+e_{l}^{\mathrm{T}}(t) e_{l}(t) \\
\left.+e_{m}^{\mathrm{T}}(t) e_{m}(t)+e_{n}^{\mathrm{T}}(t) e_{n}(t)\right) \\
+\frac{1}{2} \sum_{i=1}^{4} \frac{1}{\varepsilon_{i}}\left(k_{i}-k\right)^{2}
\end{aligned}
$$

where $k>0$ is a positive constant.

Then the time derivation of the Lyapunov function along the trajectory of error systems is

$$
\begin{aligned}
\dot{V}(t)= & e_{1}^{\mathrm{T}}(t) \dot{e}_{1}(t)+e_{2}^{\mathrm{T}}(t) \dot{e}_{2}(t)+e_{3}^{\mathrm{T}}(t) \dot{e}_{3}(t) \\
& +e_{4}^{\mathrm{T}}(t) \dot{e}_{4}(t)+e_{\alpha}^{\mathrm{T}}(t) \dot{e}_{\alpha}(t)+e_{\beta}^{\mathrm{T}}(t) \dot{e}_{\beta}(t) \\
& +e_{\gamma}^{\mathrm{T}}(t) \dot{e}_{\gamma}(t)+e_{\theta}^{\mathrm{T}}(t) \dot{e}_{\theta}(t)+e_{l}^{\mathrm{T}}(t) \dot{e}_{l}(t) \\
& +e_{m}^{\mathrm{T}}(t) \dot{e}_{m}(t)+e_{n}^{\mathrm{T}}(t) \dot{e}_{n}(t) \\
& +\dot{k}_{i} \sum_{i=1}^{4} \frac{1}{\varepsilon_{i}}\left(k_{i}-k\right)^{2} .
\end{aligned}
$$

Constituting (48), (49), (50), and (51) into (53), we get the following:

$$
\dot{V}(t)=-k\left(e_{1}^{\mathrm{T}}(t) e_{1}+e_{2}^{\mathrm{T}}(t) e_{2}+e_{3}^{\mathrm{T}}(t) e_{3}+e_{4}^{\mathrm{T}}(t) e_{4}\right)<0 .
$$

$V(t)$ is positive definite and $\dot{V}(t)<0$; thus, according to Barbalat's Lemma, MFPLS between the drive system (46) and the response system (47) is achieved. The uncertain parameters and control gain strengths can be identified and defined as well.

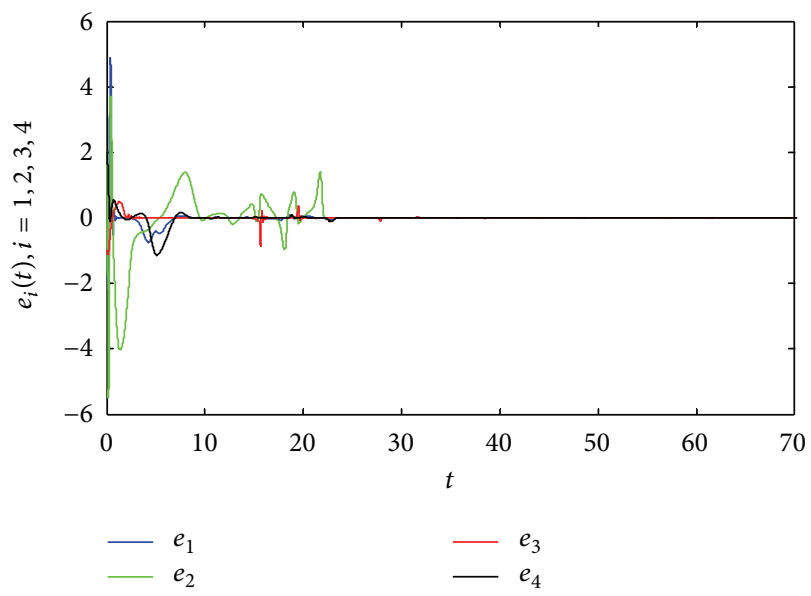

FIGURE 8: Time evolution of MFPLS errors between systems (46) and (47).

In the numerical simulation, the system parameters are selected as $\alpha=1, \beta=1.5, \gamma=26, \theta=0.7, l=10, m=28$, $n=8 / 3$, such that the drive system (46) and the response system (47) are chaotic without control. We take that the initial conditions of the drive system (46) and the response system $(47)$ are $\mathbf{x}(0)=(-2,1,4,2)^{\mathrm{T}}, \mathbf{y}(0)=(2,-2,3,4)^{\mathrm{T}}$, respectively. The constants are $\varepsilon_{1}=\varepsilon_{2}=\varepsilon_{3}=\varepsilon_{4}=10$, and the initial values of the control gain strengths are chosen as $k_{1}(0)=k_{2}(0)=k_{3}(0)=k_{4}(0)=8$. The initial values of the unknown parameters are set as $\widehat{\alpha}(0)=\widehat{\beta}(0)=\widehat{\gamma}(0)=\widehat{\theta}(0)=$ $\widehat{l}_{1}(0)=\widehat{m}_{1}(0)=\widehat{n}_{1}(0)=0.001$. The time delays are randomly selected as $\left(\tau_{1}, \tau_{2}, \tau_{3}, \tau_{4}\right)=(0.2,0.1,0.3,0.1)$. The scaling constants are taken as $\left(m_{1}, m_{2}, m_{3}, m_{4}\right)=(2,-3,-2,3)$ and nonzero continuous differentiable function is chosen as $h(t)=2 \sin (0.5 t)+1$.

Corresponding simulation results are displayed in Figures $8-11$. Figure 8 shows that the error variables $e_{1}, e_{2}, e_{3}, e_{4}$ tend to zero, respectively. Figures 9 and 10 illustrate that the estimated values of the unknown parameters approach to $\alpha=1, \beta=1.5, \gamma=26, \theta=0.7, l=10, m=28, n=8 / 3$ as $t \rightarrow \infty$, respectively. Figure 11 depicts that the control gain strength $k_{1}, k_{2}, k_{3}$, and $k_{4}$ tend to some certain value, $k_{1}=$ $32.42, k_{2}=121.3, k_{3}=12.24, k_{4}=12.62$ as $t \rightarrow \infty$. As shown in Figures 8-11, MFPLS between Lorenz-Stenflo (LS) hyperchaotic system (46) and the three-dimensional Lorenz system (47) is obtained and all the uncertain parameters are identified successfully by using the controller (49) and the parameter update rule (50). At the meantime, the control gain strengths can be estimated.

4.4. Case 4: MFPLS between a Novel Three-Dimensional Chaotic System and a Five-Dimensional Hyperchaotic System. In this subsection, MFPLS between a novel threedimensional chaotic system and a five-dimensional hyperchaotic system is analyzed.

Recently, Wu and Li [34] introduced a novel three-dimensional autonomous chaotic system by adding a quadratic cross-product term to the first equation and modifying the state variable in the third equation of a chaotic system 


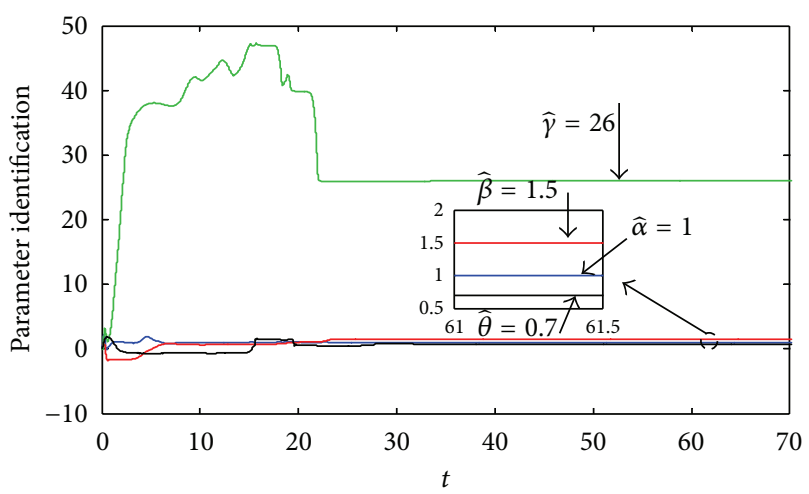

Figure 9: Time evolution of parameter estimation for the drive system (46).

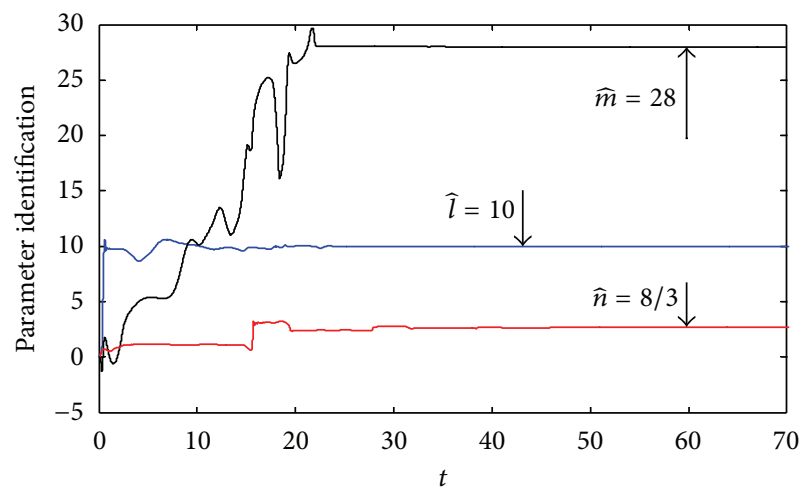

FIGURE 10: Time evolution of parameter estimation for the response system (47).

proposed by Cai et al. [35], investigated some basic dynamical properties, such as Lyapunov exponent spectrum, bifurcations, equilibria, and chaotic dynamical behaviors of the new chaotic system, and studied hybrid function projective synchronization (HFPS) of the new chaotic system. The system is described by

$$
\begin{gathered}
\dot{x}_{1}=a\left(x_{2}-x_{1}\right)+x_{2} x_{3}, \\
\dot{x}_{2}=b x_{1}+c x_{2}-x_{1} x_{3}, \\
\dot{x}_{3}=x_{2}^{2}-d x_{3},
\end{gathered}
$$

where $x_{1}, x_{2}$, and $x_{3}$ are state variables and $a, b, c$, and $d$ are the uncertain system parameters to be estimated. When $a=20, b=5, c=10, d \in[0,+\infty)$, the system behaves as hyperchaos.

The response system is a five-dimensional hyperchaotic Lorenz system, which is given by

$$
\begin{gathered}
\dot{y}_{1}=-\sigma y_{1}+\sigma y_{2}+y_{4}+u_{1}, \\
\dot{y}_{2}=r y_{1}-y_{2}-y_{1} y_{3}-y_{5}+u_{2}, \\
\dot{y}_{3}=-\beta y_{3}+y_{1} y_{2}+u_{3}, \\
\dot{y}_{4}=-y_{1} y_{3}+d_{1} y_{4}+u_{4}, \\
\dot{y}_{5}=d_{2} y_{2}+u_{5},
\end{gathered}
$$

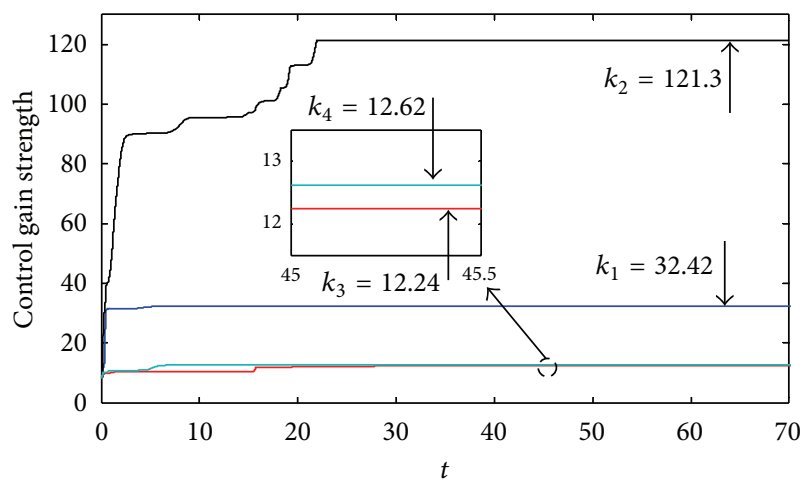

FIGURE 11: Time evolution of the control gain strength.

where $y_{1}, y_{2}, y_{3}, y_{4}$, and $y_{5}$ are state variables and $\sigma, \beta, r, d_{1}$, and $d_{2}$ are the unknown system parameters to be identified. When $\sigma=10, \beta=8 / 3, r=28, d_{1}=2$, and $d_{2} \in(2,12)$, the system is hyperchaotic with three positive LEs. $u_{1}, u_{2}, u_{3}, u_{4}$, and $u_{5}$ are the controllers to be designed.

Based on the above method, we construct two auxiliary state variable $x_{4}=x_{1}, x_{5}=x_{2}+x_{3}$. Then the drive system can be written as follows:

$$
\begin{gathered}
\dot{x}_{1}=a\left(x_{2}-x_{1}\right)+x_{2} x_{3}, \\
\dot{x}_{2}=b x_{1}+c x_{2}-x_{1} x_{3}, \\
\dot{x}_{3}=x_{2}^{2}-d x_{3}, \\
\dot{x}_{4}=a\left(x_{2}-x_{1}\right)+x_{2} x_{3}, \\
\dot{x}_{5}=b x_{1}+c x_{2}-x_{1} x_{3}+x_{2}^{2}-d x_{3} .
\end{gathered}
$$

The MFPLS error is

$$
\begin{aligned}
& e_{1}(t)=y_{1}(t)-m_{1} h(t) x_{1}\left(t-\tau_{1}\right), \\
& e_{2}(t)=y_{2}(t)-m_{2} h(t) x_{2}\left(t-\tau_{2}\right), \\
& e_{3}(t)=y_{3}(t)-m_{3} h(t) x_{3}\left(t-\tau_{3}\right), \\
& e_{4}(t)=y_{4}(t)-m_{4} h(t) x_{4}\left(t-\tau_{4}\right), \\
& e_{5}(t)=y_{5}(t)-m_{5} h(t) x_{5}\left(t-\tau_{5}\right),
\end{aligned}
$$

where $m_{i}(i=1,2,3,4,5)$ is a scaling constant, $h(t)$ is a nonzero continuous differentiable function, and the time delay vector is $\boldsymbol{\tau}=\left(\tau_{1}, \tau_{2}, \tau_{3}, \tau_{4}, \tau_{5}\right)^{\mathrm{T}}$.

Based on Theorem 3, the controller, parameter update rules, and control gain strength adapt laws are chosen as follows:

$$
\begin{aligned}
u_{1}(t)= & \widehat{\sigma}\left(y_{1}(t)-y_{2}(t)\right)-y_{4}(t)+m_{1} \dot{h}(t) x_{1}\left(t-\tau_{1}\right) \\
& +m_{1} h(t) \hat{a}\left(x_{2}\left(t-\tau_{1}\right)-x_{1}\left(t-\tau_{1}\right)\right) \\
& +m_{1} h(t) x_{2}\left(t-\tau_{1}\right) x_{3}\left(t-\tau_{1}\right)-k_{1} e_{1}
\end{aligned}
$$




$$
\begin{aligned}
& u_{2}(t)=-\widehat{r} y_{1}(t)+y_{2}(t)+y_{1}(t) y_{3}(t)+y_{5}(t) \\
& +m_{2} \dot{h}(t) x_{2}\left(t-\tau_{2}\right)-m_{2} h(t) \\
& \times\left(-\widehat{b} x_{1}\left(t-\tau_{2}\right)-\widehat{c} x_{2}\left(t-\tau_{2}\right)+x_{1}\left(t-\tau_{2}\right) x_{3}\left(t-\tau_{2}\right)\right) \\
& -k_{2} e_{2} \\
& u_{3}(t)=\widehat{\beta} y_{3}(t)-y_{1}(t) y_{2}(t)+m_{3} \dot{h}(t) x_{3}\left(t-\tau_{3}\right) \\
& -m_{3} h(t)\left(-x_{2}^{2}\left(t-\tau_{3}\right)+\widehat{d} x_{3}\left(t-\tau_{3}\right)\right)-k_{3} e_{3}, \\
& u_{4}(t)=y_{1}(t) y_{3}(t)-\widehat{d}_{1} y_{4}(t)+m_{4} \dot{h}(t) x_{4}\left(t-\tau_{4}\right) \\
& +m_{4} h(t) \hat{a}\left(x_{2}\left(t-\tau_{4}\right)-x_{1}\left(t-\tau_{4}\right)\right) \\
& +m_{4} h(t) x_{2}\left(t-\tau_{4}\right) x_{3}\left(t-\tau_{4}\right)-k_{4} e_{4}, \\
& u_{5}(t)=-\widehat{d}_{2} y_{2}(t)+m_{5} \dot{h}(t) x_{5}\left(t-\tau_{5}\right)-m_{5} h(t) \\
& \times\left(-\widehat{b} x_{1}\left(t-\tau_{5}\right)-\widehat{c} x_{2}\left(t-\tau_{5}\right)\right. \\
& +x_{1}\left(t-\tau_{5}\right) x_{3}\left(t-\tau_{5}\right) \\
& \left.-x_{2}^{2}\left(t-\tau_{5}\right)+\widehat{d} x_{3}\left(t-\tau_{5}\right)\right)-k_{5} e_{5} \text {, } \\
& \dot{e}_{a}=\dot{\hat{a}}=-e_{1} m_{1} h(t)\left(x_{2}\left(t-\tau_{1}\right)-x_{1}\left(t-\tau_{1}\right)\right) \\
& -m_{4} h(t) e_{4}\left(x_{2}\left(t-\tau_{4}\right)-x_{1}\left(t-\tau_{4}\right)\right), \\
& \dot{e}_{b}=\dot{\hat{b}}=-e_{2} m_{2} h(t) x_{1}\left(t-\tau_{2}\right)-m_{5} h(t) e_{5} x_{1}\left(t-\tau_{5}\right) \text {, } \\
& \dot{e}_{c}=\dot{\widehat{c}}=-m_{2} h(t) e_{2} x_{2}\left(t-\tau_{2}\right)-m_{5} h(t) e_{5} x_{2}\left(t-\tau_{5}\right) \text {, } \\
& \dot{e}_{d}=\dot{\vec{d}}=m_{3} h(t) e_{3} x_{3}\left(t-\tau_{3}\right)+m_{5} h(t) e_{5} x_{3}\left(t-\tau_{5}\right) \text {, } \\
& \dot{e}_{\sigma}=\dot{\hat{\sigma}}=-e_{1}\left(y_{1}(t)-y_{2}(t)\right) \text {, } \\
& \dot{e}_{\beta}=\dot{\hat{\beta}}=-e_{3} y_{3}(t) \text {, } \\
& \dot{e}_{r}=\dot{\hat{r}}=e_{2} y_{1}(t) \text {, } \\
& \dot{e}_{d_{1}}=\dot{\vec{d}}_{1}=e_{4} y_{4}(t), \\
& \dot{e}_{d_{2}}=\dot{\widehat{d}}_{2}=e_{5} y_{2}(t), \\
& \dot{k}_{i}=\varepsilon_{i} e_{i}^{2}, \quad \varepsilon_{i}>0, \quad i=1,2, \ldots, 5,
\end{aligned}
$$

where $\widehat{a}, \widehat{b}, \widehat{c}, \widehat{d}, \widehat{\sigma}, \widehat{\beta}, \widehat{r}, \widehat{d}_{1}$, and $\widehat{d}_{2}$ are the estimated values of $a, b, c, d, \sigma, \beta, r, d_{1}$, and $d_{2}$, respectively and $e_{a}=\widehat{a}-a$, $e_{b}=\widehat{b}-b, e_{c}=\widehat{c}-c, e_{d}=\widehat{d}-d, e_{\sigma}=\widehat{\sigma}-\sigma, e_{\beta}=\widehat{\beta}-\beta$, $e_{r}=\widehat{r}-r, e_{d_{1}}=\widehat{d}_{1}-d_{1}, e_{d_{2}}=\widehat{d}_{2}-d_{2}$ are parameter errors.

Proof. The Lyapunov function is chosen as

$$
\begin{aligned}
& V(t)=\frac{1}{2}\left(e_{1}^{\mathrm{T}}(t) e_{1}(t)+e_{2}^{\mathrm{T}}(t) e_{2}(t)+e_{3}^{\mathrm{T}}(t) e_{3}(t)\right. \\
& \quad+e_{4}^{\mathrm{T}}(t) e_{4}(t)+e_{5}^{\mathrm{T}}(t) e_{5}(t)+e_{a}^{\mathrm{T}}(t) e_{a}(t)
\end{aligned}
$$

$$
\begin{aligned}
& +e_{b}^{\mathrm{T}}(t) e_{b}(t)+e_{c}^{\mathrm{T}}(t) e_{c}(t)+e_{d}^{\mathrm{T}}(t) e_{d}(t) \\
& +e_{\sigma}^{\mathrm{T}}(t) e_{\sigma}(t)+e_{\beta}^{\mathrm{T}}(t) e_{\beta}(t)+e_{r}^{\mathrm{T}}(t) e_{r}(t) \\
& \left.+e_{d_{1}}^{\mathrm{T}}(t) e_{d_{1}}(t)+e_{d_{2}}^{\mathrm{T}}(t) e_{d_{2}}(t)\right) \\
& +\frac{1}{2} \sum_{i=1}^{5} \frac{1}{\varepsilon_{i}}\left(k_{i}-k\right)^{2},
\end{aligned}
$$

where $k>0$ is a positive constant.

Then the time derivation of the Lyapunov function along the trajectory of error systems is

$$
\begin{aligned}
\dot{V}(t)= & e_{1}^{\mathrm{T}}(t) \dot{e}_{1}(t)+e_{2}^{\mathrm{T}}(t) \dot{e}_{2}(t)+e_{3}^{\mathrm{T}}(t) \dot{e}_{3}(t) \\
& +e_{4}^{\mathrm{T}}(t) \dot{e}_{4}(t)+e_{5}^{\mathrm{T}}(t) \dot{e}_{5}(t)+e_{a}^{\mathrm{T}}(t) \dot{e}_{a}(t) \\
& +e_{b}^{\mathrm{T}}(t) \dot{e}_{b}(t)+e_{c}^{\mathrm{T}}(t) \dot{e}_{c}(t)+e_{d}^{\mathrm{T}}(t) \dot{e}_{d}(t) \\
& +e_{\sigma}^{\mathrm{T}}(t) \dot{e}_{\sigma}(t)+e_{\beta}^{\mathrm{T}}(t) \dot{e}_{\beta}(t)+e_{r}^{\mathrm{T}}(t) \dot{e}_{r}(t) \\
& +e_{d_{1}}^{\mathrm{T}}(t) \dot{e}_{d_{1}}(t)+e_{d_{2}}^{\mathrm{T}}(t) \dot{e}_{d_{2}}(t) \\
& +\dot{k}_{i} \sum_{i=1}^{5} \frac{1}{\varepsilon_{i}}\left(k_{i}-k\right)^{2} .
\end{aligned}
$$

Constituting (58) and (59) into (61), we get the following:

$$
\begin{aligned}
\dot{V}(t) & =-k\left(e_{1}^{\mathrm{T}}(t) e_{1}+e_{2}^{\mathrm{T}}(t) e_{2}+e_{3}^{\mathrm{T}}(t) e_{3}+e_{4}^{\mathrm{T}}(t) e_{4}+e_{5}^{\mathrm{T}}(t) e_{5}\right) \\
& <0 .
\end{aligned}
$$

$V(t)$ is positively definite and $\dot{V}(t)<0$; thus, according to Barbalat's Lemma, MFPLS between the drive system (57) and the response system (56) is achieved. The uncertain parameters and control gain strengths can be identified and defined as well.

In the simulation, the system parameters are selected as $a=20, b=5, c=10, d=2, \sigma=10, \beta=8 / 3, r=28, d_{1}=2$, and $d_{2}=8$, such that the drive system (57) and the response system (56) can exhibit chaotic behaviors without control. We assume that the initial conditions of the drive system (57) and the response system $(56)$ as $\mathbf{x}(0)=(-2,1,4,2,-3)^{\mathrm{T}}$, $\mathbf{y}(0)=(2,-2,3,6,1)^{\mathrm{T}}$, respectively. We choose the initial values of the unknown parameters as $\widehat{a}(0)=\widehat{b}(0)=\widehat{c}(0)=$ $\widehat{d}(0)=\widehat{\sigma}(0)=\widehat{\beta}(0)=\widehat{r}(0)=\widehat{d}_{1}(0)=\widehat{d}_{2}(0)=0.001$. We select the initial values of the control gain strength as $k_{1}(0)=$ $k_{2}(0)=k_{3}(0)=k_{4}(0)=k_{5}(0)=4$, and the constants as $\varepsilon_{1}=\varepsilon_{2}=\varepsilon_{3}=\varepsilon_{4}=\varepsilon_{5}=6$. The time delay is arbitrarily chosen as $\left(\tau_{1}, \tau_{2}, \tau_{3}, \tau_{4}, \tau_{5}\right)=(0.3,0.4,0.2,0.1,0.2)$. We take the scaling constants as $\left(m_{1}, m_{2}, m_{3}, m_{4}, m_{5}\right)=(-2,3,-1,2,-5)$ and nonzero continuous differentiable function as $h(t)=$ $\sin (t)$. The corresponding simulation results are illustrated in Figures 12-15.

Figure 12 shows that the synchronization errors converge to zero after a transient time, which indicates that MFPLS between the drive system (57) and the response system (56) 


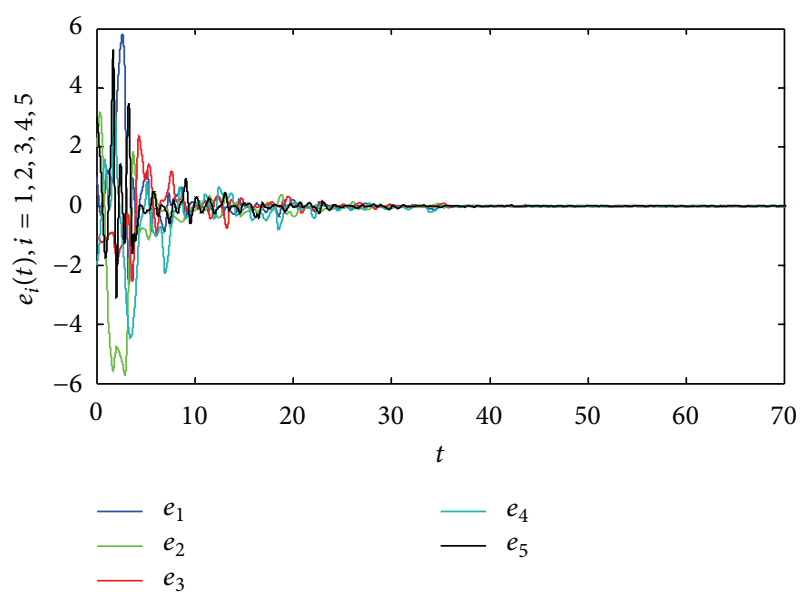

FIGURE 12: Time evolution of MFPLS errors between the system (57) and the system (56).

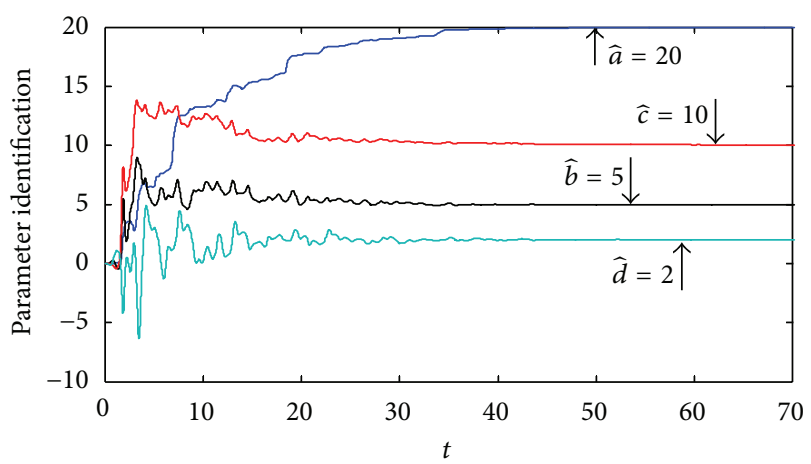

FIgURE 13: Time evolution of parameter estimation for the drive system (57).

is achieved. From Figures 13 and 14, it is easy to see that the nine unknown parameters are also identified. Time evolution of the adaptive control strength is illustrated in Figure 15, and the control strengths approach to $k_{1}=29.32, k_{2}=70.77$, $k_{3}=23.85, k_{4}=47.18$ and $k_{5}=25.98$ as $t \rightarrow \infty$. Hence, the MFPLS between the 3-dimensional hyperchaotic system and the 5-dimensional hyperchaotic system are attained, all the uncertain parameters can be estimated and the control gain strengths can be given, too.

\section{Conclusions}

The paper investigated MFPLS of hyperchaotic or chaotic system, when the system parameters are all uncertain and the dimension and structure of the drive system and the response system are the same or different. A general theorem for controller designing, parameter update rule designing, and control gain strength adapt law designing is introduced by using adaptive control method, and it has been proven effective theoretically based on Lyapunov stability theory. It is worth mentioning that the systems can have the identical or different dimensions and structures, and the control gain strengths can be identified adaptively.

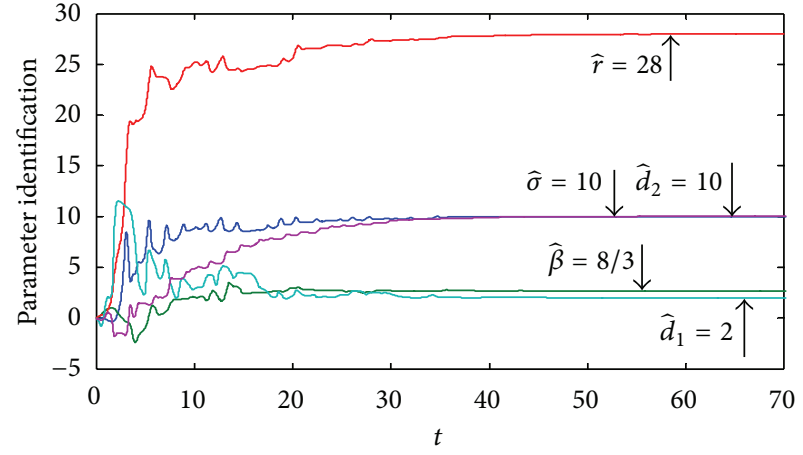

FIGURE 14: Time evolution of parameter estimation for the response system (56).

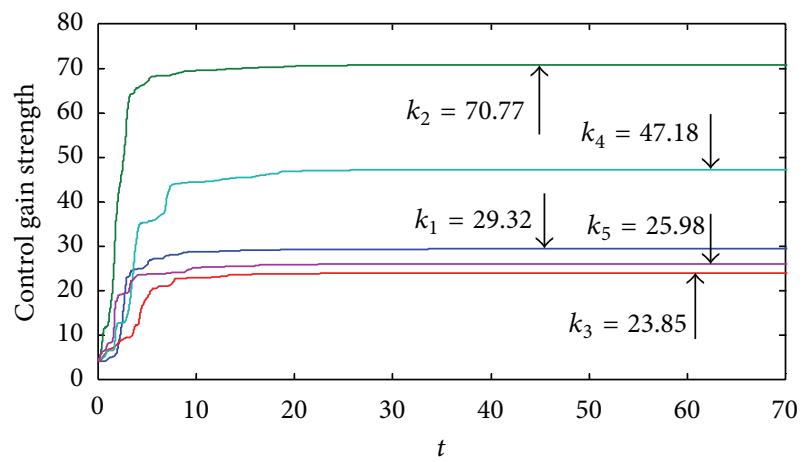

FIgURE 15: Time evolution of the control gain strength.

Furthermore, the proposed method is applied to four typical examples, which include MFPLS between two fivedimensional hyperchaotic systems with identical structures, MFPLS between two four-dimensional hyperchaotic systems with different structures, MFPLS between a four-dimensional hyperchaotic system and a three-dimensional chaotic system, and MFPLS between a novel three-dimensional chaotic system and a five-dimensional hyperchaotic system. In every case, controller, parameter update rule, and control gain strength adapt law are constructed in detail. The corresponding numerical simulations are performed to show the effectiveness of our results.

By now, many fractional-order differential systems such as fractional-order Chua's circuit, the fractional-order van der pol system, and the fractional-order Lorenz system, are chaotic. And study on the synchronization of chaotic fractional-order differential systems has greatly attracted interest of many researchers due to its potential applications in secure communication and control processing [36-38]. Wang et al. [36, 37] introduced projective synchronization of fractional-order chaotic systems based on linear separation and synchronization of fractional-order chaotic systems with activation feedback control. In [38], modified projective synchronization of fractional-order chaotic systems via active sliding mode control is analyzed, and active sliding mode controller is proposed to synchronize two different fractional-order differential systems based on the stability 
theorems of fractional-order linear system. Research on MFPLS of fractional-order chaotic systems is interesting and useful. The adding of time delay makes the solving of the equation more difficult. To the best of our knowledge, modified function projective lag synchronization of fractionalorder chaotic systems are not studied. So, we will investigate the MFPLS of fractional-order chaotic systems and derive some stability criteria in a near future.

\section{Acknowledgments}

This work is supported by the National Natural Science Foundation of China (Grants nos. 61004006 and 61203094), Natural Science Foundation of Educational Committee of Henan Province of China (Grants nos. 12A460001, 2011A520001, and 2011A520004), the joint funds between Henan Provincial Government and Ministry of Education of China (Grant no. SBGJ090603), China Postdoctoral Science Foundation (Grant no. 2013M530181), and Shanghai Postdoctoral Scientific Program (Grant no. 13R21410600).

\section{References}

[1] G. Chen and X. Dong, From Chaos to Order, World Scientific, Singapore, 1998.

[2] C. Y. Chee and D. Xu, "Secure digital communication using controlled projective synchronization of chaos," Chaos, Solitons and Fractals, vol. 23, no. 3, pp. 1063-1070, 2005.

[3] X. D. Li and X. L. Fu, "Synchronization of chaotic delayed neural networks with impulsive and stochastic perturbations," Communications in Nonlinear Science and Numerical Simulation, vol. 16, no. 2, pp. 885-894, 2011.

[4] L. M. Pecora and T. L. Carroll, "Synchronization in chaotic systems," Physical Review Letters, vol. 64, no. 8, pp. 821-824, 1990.

[5] M. M. El-Dessoky, "Anti-synchronization of four scroll attractor with fully unknown parameters," Nonlinear Analysis. Real World Applications, vol. 11, no. 2, pp. 778-783, 2010.

[6] F. A. Breve, L. Zhao, M. G. Quiles, and E. E. N. Macau, "Chaotic phase synchronization and desynchronization in an oscillator network for object selection," Neural Networks, vol. 22, no. 5-6, pp. 728-737, 2009.

[7] C. Li and X. Liao, "Lag synchronization of Rossler system and Chua circuit via a scalar signal," Physics Letters A, vol. 329, no. 4-5, pp. 301-308, 2004.

[8] G. Wen and D. Xu, "Nonlinear observer control for fullstate projective synchronization in chaotic continuous-time systems," Chaos, Solitons and Fractals, vol. 26, no. 1, pp. 71-77, 2005.

[9] K. Sebastian Sudheer and M. Sabir, "Adaptive function projective synchronization of two-cell quantum-CNN chaotic oscillators with uncertain parameters," Physics Letters A, vol. 373, no. 21, pp. 1847-1851, 2009.

[10] H. Du, Q. Zeng, C. Wang, and M. Ling, "Function projective synchronization in coupled chaotic systems," Nonlinear Analysis. Real World Applications, vol. 11, no. 2, pp. 705-712, 2010.

[11] Y. Yu and H.-X. Li, "Adaptive generalized function projective synchronization of uncertain chaotic systems," Nonlinear Analysis. Real World Applications, vol. 11, no. 4, pp. 2456-2464, 2010.
[12] X. J. Wu and H. T. Lu, "Adaptive generalized function projective lag synchronization of different chaotic systems with fully uncertain parameters," Chaos, Solitons and Fractals, vol. 44, no. 10, pp. 802-810, 2011.

[13] X.-J. Wu and H.-T. Lu, "Generalized projective lag synchronization between different hyperchaotic systems with uncertain parameters," Nonlinear Dynamics, vol. 66, no. 1-2, pp. 185-200, 2011.

[14] H. Du, Q. Zeng, and C. Wang, "Modified function projective synchronization of chaotic systems," Chaos, Solitons and Fractals, vol. 42, no. 4, pp. 2399-2404, 2009.

[15] K. Sudheer and M. Sebastian Sabir, "Modified function projective synchronization of hyperchaotic systems through OpenPlus-Closed-Loop coupling," Physics Letters A, vol. 374, no. 1920, pp. 2017-2023, 2010.

[16] K. S. Sudheer and M. Sabir, "Adaptive modified function projective synchronization between hyperchaotic Lorenz system and hyperchaotic Lu system with uncertain parameters," Physics Letters A, vol. 373, no. 41, pp. 3743-3748, 2009.

[17] K. Sudheer and M. Sebastian Sabir, "Adaptive modified function projective synchronization of multiple time-delayed chaotic Rossler system," Physics Letters A, vol. 375, no. 8, pp. 1176-1178, 2011.

[18] S. Zheng, G. Dong, and Q. Bi, "Adaptive modified function projective synchronization of hyperchaotic systems with unknown parameters," Communications in Nonlinear Science and Numerical Simulation, vol. 15, no. 11, pp. 3547-3556, 2010.

[19] J. A. Wang and H. P. Liu, "Adaptive modified function projective synchronization of different hyperchaotic systems," Acta Physica Sinica, vol. 59, no. 4, pp. 2265-2271, 2010.

[20] P. Liu, S. T. Liu, and X. Li, "Adaptive modified function projective synchronization of general uncertain chaotic complex systems," Physica Scripta, vol. 85, no. 3, Article ID 035005, 2012.

[21] Z. Y. Sun, G. Q. Si, F. H. Min, and Y. Zhang, "Adaptive modified function projective synchronization and parameter identification of uncertain hyperchaotic (chaotic) systems with identical or non-identical structures," Nonlinear Dynamics, vol. 68, no. 4, pp. 471-486, 2012.

[22] S. Zheng, "Adaptive modified function projective synchronization of unknown chaotic systems with different order," Applied Mathematics and Computation, vol. 218, no. 10, pp. 5891-5899, 2012.

[23] M. M. El-Dessoky, M. T. Yassen, and E. Saleh, "Adaptive modified function projective synchronization between two different hyperchaotic dynamical systems," Mathematical Problems in Engineering, vol. 2012, Article ID 810626, 16 pages, 2012.

[24] G. Y. Fu, "Robust adaptive modified function projective synchronization of different hyperchaotic systems subject to external disturbance," Communications in Nonlinear Science and Numerical Simulation, vol. 17, no. 6, pp. 2602-2608, 2012.

[25] H. Y. Du, Q. S. Zeng, and N. Lue, "A general method for modified function projective lag synchronization in chaotic systems," Physics Letters A, vol. 374, no. 13-14, pp. 1493-1496, 2011.

[26] G. L. Cai, P. Hu, and Y. X. Li, "Modified function lag projective synchronization of a financial hyperchaotic system," Nonlinear Dynamics, vol. 69, no. 3, pp. 1457-1464, 2012.

[27] H. Liu, H. J. Yu, and W. Xiang, "Modified function projective lag synchronization for multi-scroll chaotic system with unknown disturbance," Acta Physica Sinica, vol. 61, no. 18, Article ID 180503, 2012. 
[28] H. Y. Du, "Adaptive Open-Plus-Closed-Loop control method of modified function projective synchronization in complex networks," International Journal of Modern Physics C, vol. 22, no. 12, pp. 1393-1407, 2011.

[29] P. Haipeng, L. X. Li, Y. X. Yang, and F. Sun, "Conditions of parameter identification from time series," Physical Review E, vol. 83, no. 2, Article ID 036202, 2011.

[30] W. Yu, G. Chen, J. Cao, J. Lu, and U. Parlitz, "Parameter identification of dynamical systems from time series," Physical Review E, vol. 75, no. 6, Article ID 067201, 4 pages, 2007.

[31] G. Hu, "Generating hyperchaotic attractors with three positive Lyapunov exponents via state feedback control," International Journal of Bifurcation and Chaos, vol. 19, no. 2, pp. 651-660, 2009.

[32] A. Chen, J. Lu, J. Lü, and S. Yu, "Generating hyperchaotic Lü attractor via state feedback control," Physica A, vol. 364, pp. 103110, 2006

[33] L. Stenflo, "Generalized Lorenz equations for acoustic-gravity waves in the atmosphere," Physica Scripta, vol. 53, pp. 83-84, 1996.

[34] X. J. Wu and S. Z. Li, "Dynamics analysis and hybrid function projective synchronization of a new chaotic system," Nonlinear Dynamics, vol. 69, no. 4, pp. 1979-1994, 2012.

[35] G. Cai, Z. Tan, W. Zhou, and W. Tu, "The dynamical analysis and control of a new chaotic system," Acta Physica Sinica, vol. 56, no. 11, pp. 6230-6237, 2007.

[36] X. Y. Wang and Y. J. He, "Projective synchronization of fractional order chaotic system based on linear separation," Physics Letters A, vol. 372, no. 4, pp. 435-441, 2008.

[37] X. Y. Wang and J. M. Song, "Synchronization of the fractional order hyperchaos Lorenz systems with activation feedback control," Communications in Nonlinear Science and Numerical Simulation, vol. 14, no. 8, pp. 3351-3357, 2009.

[38] X. Y. Wang, X. P. Zhang, and C. Ma, "Modified projective synchronization of fractional-order chaotic systems via active sliding mode control," Nonlinear Dynamics, vol. 69, no. 1-2, pp. 511-517, 2012. 


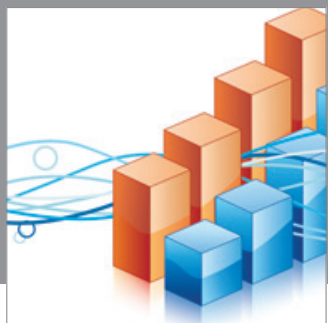

Advances in

Operations Research

mansans

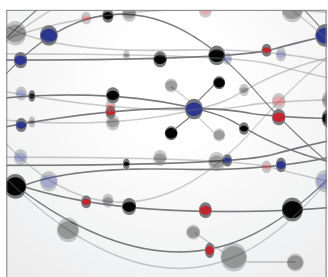

The Scientific World Journal
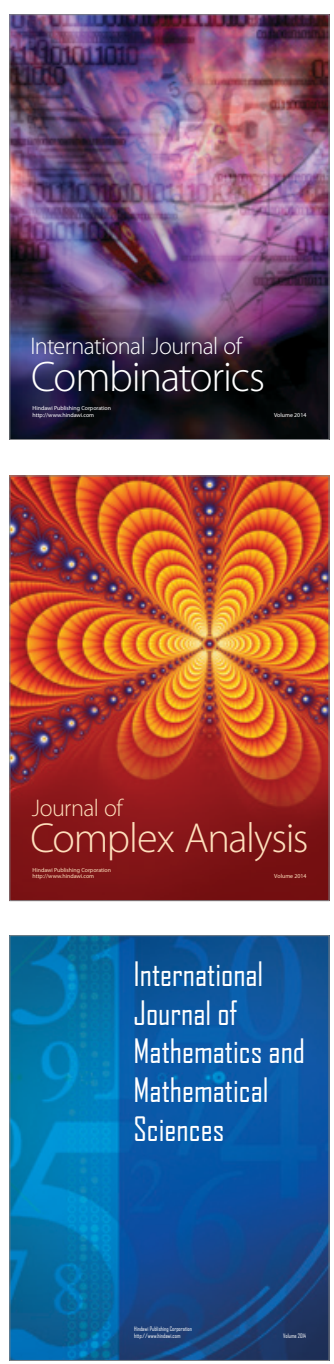
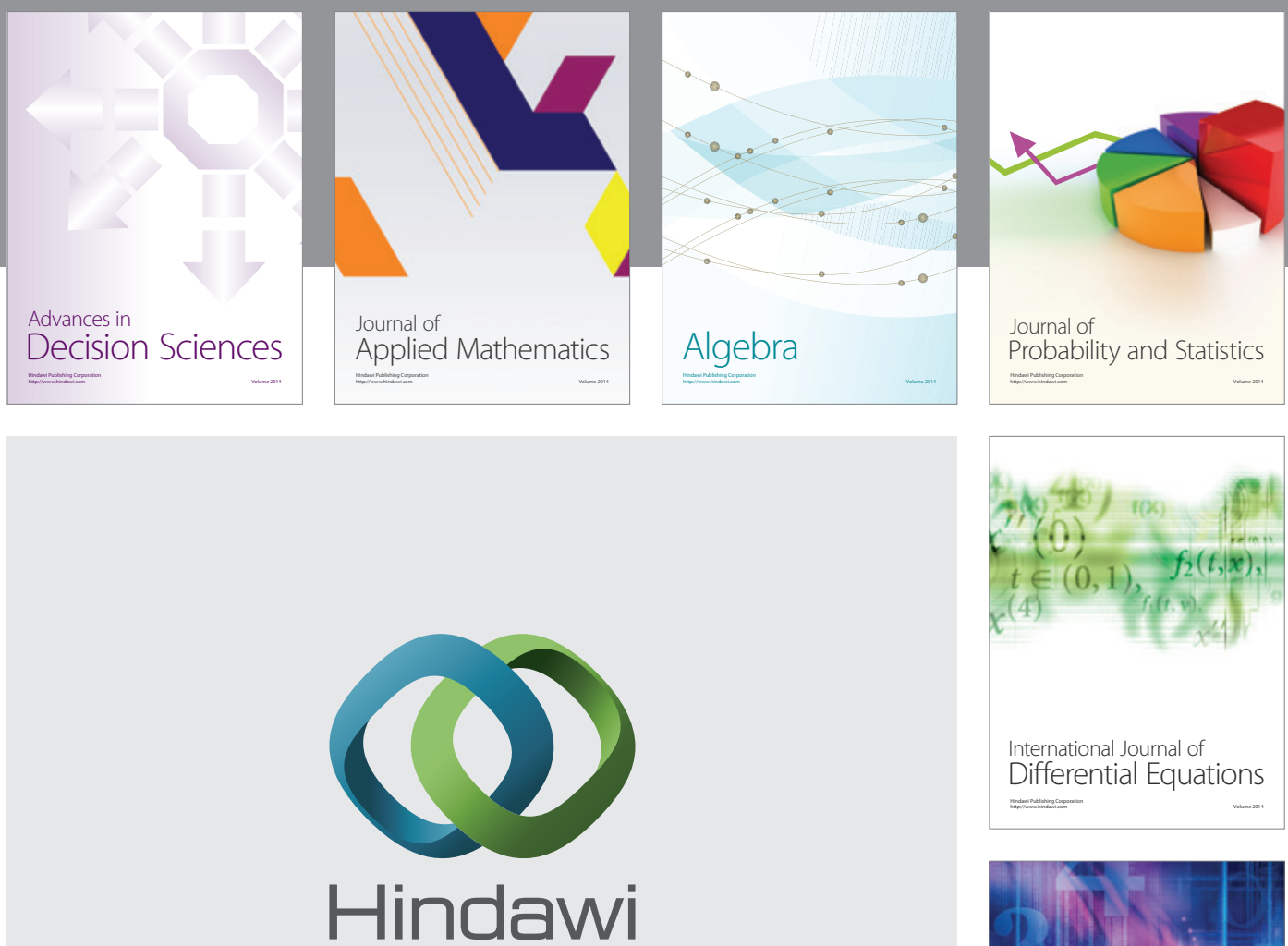

Submit your manuscripts at http://www.hindawi.com
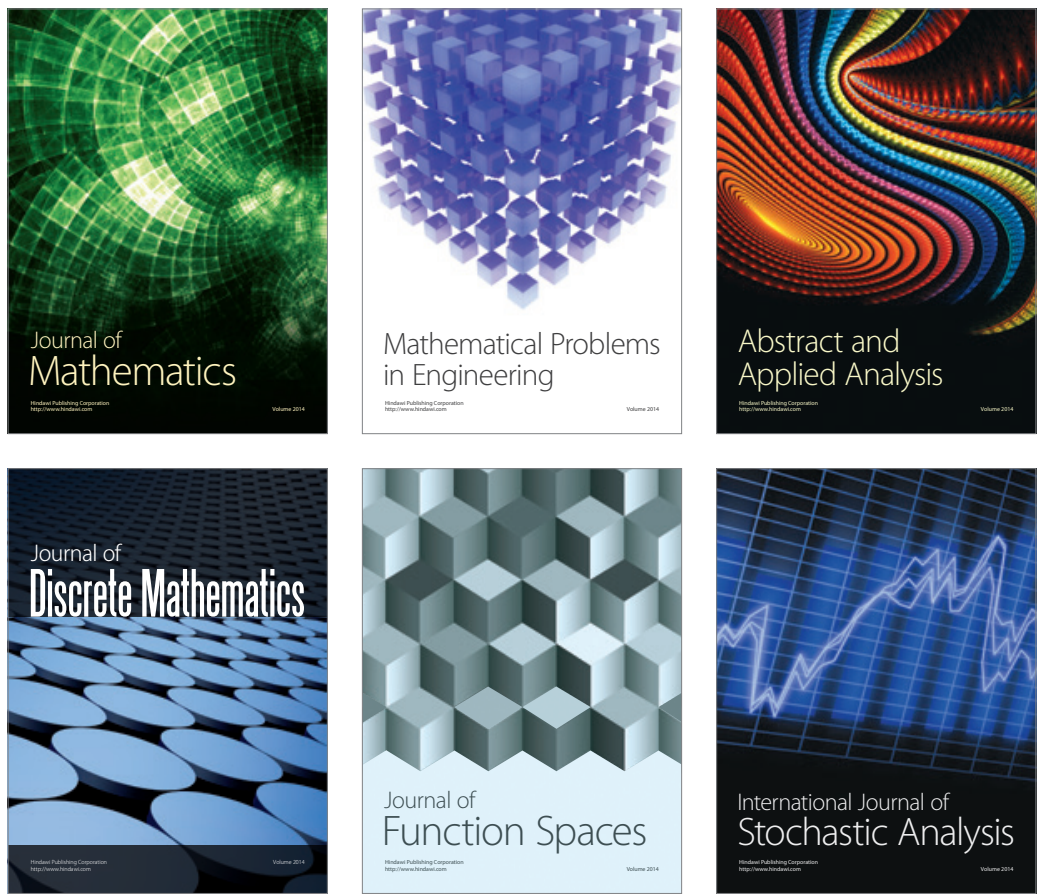

Journal of

Function Spaces

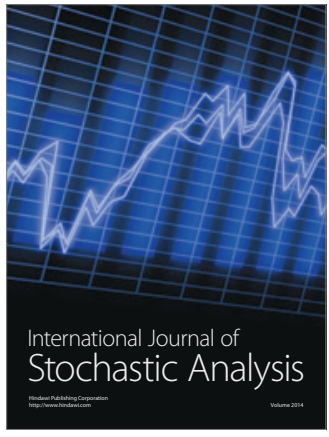

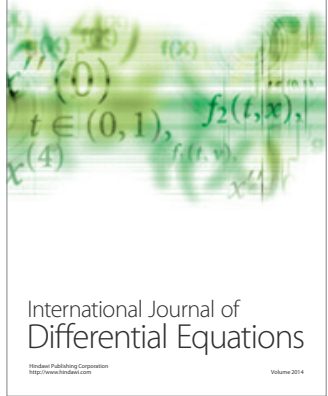
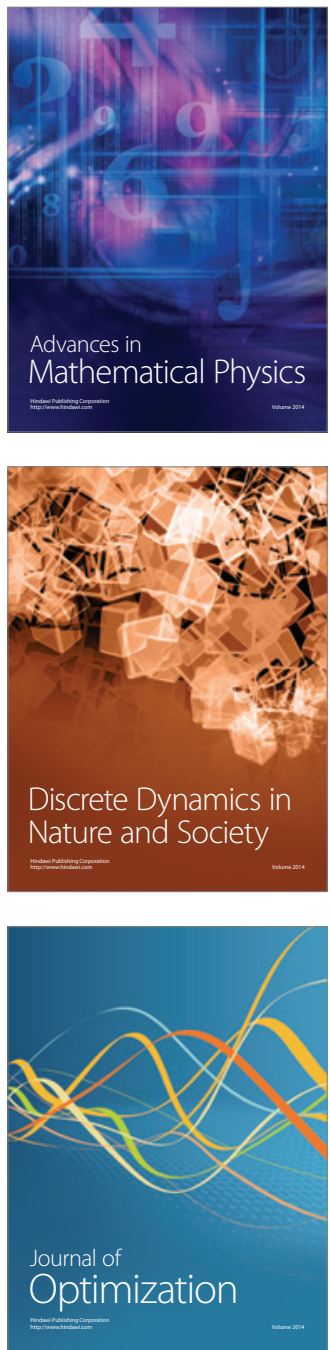